\title{
On the Introduction of an Agile, Temporary Workforce into a Tandem Queueing System
}

DAVID L. KAUFMAN

davidlk@engin.umich.edu

Department of Industrial and Operations Engineering, University of Michigan, 1205 Beal Avenue, Ann Arbor, Michigan 48109-2117

HYUN-SOO AHN

hsahn@umich.edu

Operations and Management Science, Ross School of Business, University of Michigan, 701 Tappan Street, Ann Arbor, Michigan 48109-1234

MARK E. LEWIS

melewis@orie.cornell.edu School of Operations Research and Industrial Engineering, Cornell University, 206 Rhodes Hall, Ithaca, New York 14853

Received 27 February 2004; Revised 18 May 2005

\begin{abstract}
We consider a two-station tandem queueing system where customers arrive according to a Poisson process and must receive service at both stations before leaving the system. Neither queue is equipped with dedicated servers. Instead, we consider three scenarios for the fluctuations of workforce level. In the first, a decision-maker can increase and decrease the capacity as is deemed appropriate; the unrestricted case. In the other two cases, workers arrive randomly and can be rejected or allocated to either station. In one case the number of workers can then be reduced (the controlled capacity reduction case). In the other they leave randomly (the uncontrolled capacity reduction case). All servers are capable of working collaboratively on a single job and can work at either station as long as they remain in the system. We show in each scenario that all workers should be allocated to one queue or the other (never split between queues) and that they should serve exhaustively at one of the queues depending on the direction of an inequality. This extends previous studies on flexible systems to the case where the capacity varies over time. We then show in the unrestricted case that the optimal number of workers to have in the system is non-decreasing in the number of customers in either queue.
\end{abstract}

Keywords: queues and service, scheduling theory, stochastic

AMS subject classification: 90B22, 90B36

\section{Introduction}

The use of an agile workforce is becoming more prevalent in the manufacturing and service sectors. Practitioners would like to understand the benefits of cross-training to know whether they exceed the costs. If workers or machines can perform a variety of 
tasks, they may be reallocated to alleviate areas of high congestion. Most models, however, assume that the number of workers available is predetermined. In many practical situations it is often the case that the number of workers available is dynamic. For example, consider a simple two cell factory where a company manufactures two products, $\mathrm{A}$ and B. Suppose some of the workers have the primary tasks of manufacture and inspection of product $\mathrm{A}$, but are also trained to perform the same tasks for product $\mathrm{B}$. A decision-maker monitors the workload of the entire job shop and can decide to reallocate some of the workers to either the manufacture or inspection portion of the product $\mathrm{B}$ line. This, of course, reduces the capacity to produce A and can be costly to the system. Three questions arise:

- Should cross-trained workers be assigned to work on product B?

- If they are to be assigned to product $\mathrm{B}$, should they be allocated to the manufacture or inspection station?

- How long should they remain working on the product B line?

We address these questions in three contexts; each from the point of view of the product $\mathrm{B}$ line. The first is called the unrestricted case where the decision-maker can increase or decrease capacity to produce product B at any time. In the second model, workers become available randomly but may be released by the decision-maker; controlled capacity reduction. In the final problem workers randomly become available and randomly leave the system; uncontrolled capacity reduction. We note that the decision-maker has less and less control over the availability of cross-trained workers from the first to the third problem.

To illustrate each problem, consider the availability of health care professionals (doctors, nurses, etc.) in various hospital operations. It is standard practice for a nurse or doctor who is currently assigned to work in other care units, such as the intensive care unit (ICU) or the outpatient clinic, to be called to the emergency room (ER) or trauma center to handle higher priority patients and then returned to their primary assignment when the workload has subsided. This practice is called "jeopardy". From the perspective of the other care units, the time between calls that take workers away and the time they (or some other worker) become available again can be modeled as random events; the uncontrolled capacity reduction case. On the other hand, since the ER and trauma center are usually the highest priority (and often the most costly) operation performed in a hospital, they can add and subtract workers as needed; the unrestricted case. Finally, while arrivals of workers may be random (from say the ER), it is often the case that some of these nurses are released to work in the general rounds when not needed; the controlled capacity reduction case.

Each problem is considered under the infinite horizon discounted expected cost and average expected cost criteria. Since the average case is considered, we present sufficient conditions for the stability of each model. We then show the rather surprising result that the optimal allocation policy is "independent" of the decision to increase or decrease capacity in the sense that a rule analogous to the classic $c \mu$-rule applies (no matter how 
many workers are available). Once this assertion is made, Markov decision processes are employed to prove that optimal policies in the unrestricted case are monotone in the number of customers in each queue. This result is used to develop heuristics for each case.

Of particular relation to the present work is the work of Ahn et al. $[1,2]$ where the clearing system and dynamic versions of this problem with a static number of servers are considered. In each case, the authors provide conditions under which the optimal policy is exhaustive in each station. Duenyas et al. [13] and Iravani et al. [20], in parallel, consider a tandem queueing system with one flexible server and characterize the optimal policy to be a monotone switching curve. The control of a flexible workforce in minimizing the cycle time of each job is discussed in Van Oyen et al. [26]. Andradottir et al. [3] consider the control of flexible servers to maximize throughput when the service rate is additive. Javidi et al. [21] consider two interconnected queues with identical machines and derive sufficient conditions under which the policy that prioritizes jobs in queue 1 minimizes the expected value of the first time that the system becomes empty (i.e., the dynamic version of makespan). Other related works include the work on "bucket brigades" by Bartholdi and Eisenstein [5] and Bartholdi et al. [4,6].

The scheduling of (temporary) workers in queueing systems is closely related to the scheduling of a removable server. Early work in this area considered the question of when to schedule the server to be offline and online (cf. $[19,27])$. The authors show the existence of control limits determining when the server should be turned on and off. More recently, Feinberg and Kella [16] show the optimality of " $D$-policies" that turn the server off when the system empties and turn it back on when the workload reaches some level $D$. There has also been a considerable amount of work on service rate control in queueing systems. Classic models include the work of Crabill [10], where it is shown that the optimal service rate is non-decreasing in the number of customers in the system. More recent studies of service rate control problems include the work of Stidham and Weber [25] and George and Harrison [17]. In each case, the authors consider models analogous to our unrestricted case but with the cost function non-decreasing in the one-dimensional state. No such representation holds here. Other related works include $[14,15]$.

The rest of the paper is organized as follows. Section 2 contains formal definitions of the problems considered and Markov decision process formulations. In the same section, we state results leading to the stability of the network and the existence of a solution to the average cost optimality equations. The main results of the paper are contained in Sections 3 and 4; the optimality of an exhaustive policy in each case and the monotone structure of an optimal policy in the unrestricted case. Some examples displaying a significant difference between the models are provided in Section 5. Promising heuristics are also introduced and analyzed. We conclude the paper in Section 6.

\section{Preliminaries and model formulation}

Consider a two station, tandem queueing system where customer arrivals to station 1 follow a Poisson process of rate $\lambda>0$. After receiving service at station 1 , customers 
proceed immediately to station 2 and must receive service in order to leave the system. The service requirement for all customers is exponential with (finite) means $1 / \mu_{1}$ and $1 / \mu_{2}$ for stations 1 and 2, respectively. Neither station is equipped with a dedicated, permanent server. Instead, in the unrestricted case the number of workers may be increased or decreased, while in the controlled or uncontrolled capacity reduction cases workers arrive according to a Poisson process at rate $\alpha(k)>0$ when there are $k$ workers currently in the system. In the uncontrolled capacity reduction case, accepted workers are available for an exponential amount of time with rate $\gamma$.

Once the decision is made to increase the capacity, workers are immediately assigned to a station. Assume that all workers are identical and able to work at rate 1 regardless of the station to which they are assigned. Note that this along with the assumption that the service requirements are exponentially distributed implies that the time to complete a job at station $i$ is exponential with rate $\mu_{i}$. Assume that customers in service can be preempted to reallocate workers and that when more than one worker is working at a station their rates are additive; servers can collaborate on a single job. Let $\ell<\infty$ represent the maximum number of workers that can be in the system at any particular time or equivalently set $\alpha(k)=0$ for $k \geq \ell$.

Define $h_{i}, i=1,2$, to be the rate at which holding costs are accrued for each customer at station $i$ and $r(k)$ to be the (finite, real-valued) cost rate of having $k$ workers in the system. Intuitively, this is the opportunity cost of having the workers allocated to this station instead of in some other part of the job shop described in Section 1. We assume that $r(0)=0$. At each customer arrival and departure time, the decision-maker must decide how to allocate the flexible workers to each station. In the unrestricted case, the decision-maker must decide how many workers to keep (or add) for the coming period. In the controlled capacity reduction case, the decision-maker can reduce the number of workers at any time, while in the uncontrolled capacity reduction case it must wait for workers to leave. In the controlled and uncontrolled capacity reduction cases, workers can only be added when the opportunity arises. A decision must then be made if they should be rejected. In either case, workers that are rejected are lost forever.

For the remainder of the paper we differentiate the unrestricted, controlled and uncontrolled capacity reduction cases with superscripts $U, C$, and $F$, respectively. Unless otherwise specified, quantities without superscripts have a common definition for each problem. In each scenario a decision rule, say $d(x)$, is a function that maps the state space to the set of potential actions (the action space). A sequence of decision rules is called a policy and prescribes what action should be taken for each state at any particular time. Note that although we have defined policies to only cover those that are Markovian, this set is sufficient to guarantee the optimality over the larger set of non-anticipating policies. Let $\mathbb{X}^{U} \equiv \mathbb{Z}^{+} \times \mathbb{Z}^{+}$and $\mathbb{X}^{C}=\mathbb{X}^{F} \equiv \mathbb{X}^{U} \times\{0,1, \ldots, \ell\}$ denote the respective state spaces of each problem, where $\mathbb{Z}^{+}$is the set of non-negative integers. The first two elements of the state space represent the number of customers at each station and the last element (in $\mathbb{X}^{C}$ and $\mathbb{X}^{F}$ ) is the number of workers available to be allocated to 
either station. Let $\Pi$ denote the set of non-anticipating, non-idling policies and suppose for a fixed $\pi \in \Pi$ and $t \geq 0$ that $Q_{1}^{\pi}(t), Q_{2}^{\pi}(t)$ and $Z^{\pi}(t)$ represent the queue length processes for station 1 and 2 and number of workers available at time $t$, respectively. Define

$$
\begin{aligned}
v_{t, \theta}^{\pi}(x) & \equiv \mathbb{E}_{x} \int_{0}^{t} e^{-\theta s}\left[h_{1} Q_{1}^{\pi}(s)+h_{2} Q_{2}^{\pi}(s)+r\left(Z^{\pi}(s)\right)\right] d s, \\
v_{\theta}^{\pi}(x) & \equiv \lim _{t \rightarrow \infty} v_{t, \theta}^{\pi}(x), \\
\rho^{\pi}(x) & \equiv \limsup _{t \rightarrow \infty} \frac{v_{t, 0}^{\pi}(x)}{t},
\end{aligned}
$$

where $\theta \geq 0$ is the discount factor. The equations (2.1)-(2.3) define the finite horizon expected discounted cost, the infinite horizon expected discounted cost, and the longrun average expected cost, respectively. In the finite horizon case, only the portion of the policy required for the time horizon is used. In each case we define the optimal values

$$
\begin{aligned}
v_{t, \theta}(x) & =\inf _{\pi \in \Pi} v_{t, \theta}^{\pi}(x), \\
v_{\theta}(x) & =\inf _{\pi \in \Pi} v_{\theta}^{\pi}(x), \\
\rho(x) & =\inf _{\pi \in \Pi} \rho^{\pi}(x),
\end{aligned}
$$

where a policy that achieves the infimum of any of the respective criteria is deemed optimal.

We will find it instructive to consider both the continuous-time problem presented and its discrete-time analogue. In the discrete-time case assume that uniformization in the spirit of Lippman [22] has been applied so that the optimal discounted and average costs are scaled by a constant. The optimal decisions are unaffected. Let $\Psi=\lambda+\max _{k \in\{0,1, \ldots, \ell\}}\{\alpha(k)\}+\ell\left(\max \left\{\mu_{1}, \mu_{2}\right\}+\gamma\right)$ be the uniformization constant and $\beta=\frac{\Psi}{\theta+\Psi}$ be the scaled discount factor. Without loss of generality assume that $\Psi=1$.

In order to simplify notation, for each decision-making scenario, let $g$ be any realvalued function on the respective state space. Define the following mappings for the unrestricted model

$$
\begin{aligned}
H_{\beta}^{U} g(i, j)= & \min _{k \in\{0,1, \ldots, \ell\}}\left\{r(k)+\beta\left(\lambda g(i+1, j)+\min _{0 \leq x \leq k}\left\{x \mu_{1} g\left((i-1)^{+}, j+1\right)\right.\right.\right. \\
& \left.\left.\left.+(k-x) \mu_{2} g\left(i,(j-1)^{+}\right)+\left[1-\left(\lambda+x \mu_{1}+(k-x) \mu_{2}\right)\right] g(i, j)\right\}\right)\right\},
\end{aligned}
$$


for the uncontrolled capacity reduction model

$$
\begin{aligned}
H_{\beta}^{F} g(i, j, k)= & r(k)+\beta(\lambda g(i+1, j, k)+\gamma k g(i, j, k-1) \\
& +\alpha(k) \min \{g(i, j, k), g(i, j,(k+1) \wedge \ell)\} \\
& +\min _{0 \leq x \leq k}\left\{x \mu_{1} g\left((i-1)^{+}, j+1, k\right)+(k-x) \mu_{2} g\left(i,(j-1)^{+}, k\right)\right. \\
& \left.\left.+\left[1-\left(\lambda+k \gamma+\alpha(k)+x \mu_{1}+(k-x) \mu_{2}\right)\right] g(i, j, k)\right\}\right),
\end{aligned}
$$

and for the controlled capacity reduction model

$$
\begin{aligned}
H_{\beta}^{C} g(i, j, k)= & \min _{0 \leq m \leq k}\{r(m)+\beta(\lambda g(i+1, j, m) \\
& +\alpha(m) \min \{g(i, j, m), g(i, j,(m+1) \wedge \ell)\} \\
& +\min _{0 \leq x \leq m}\left\{x \mu_{1} g\left((i-1)^{+}, j+1, m\right)+(m-x) \mu_{2} g\left(i,(j-1)^{+}, m\right)\right. \\
& \left.\left.\left.+\left[1-\left(\lambda+\alpha(m)+x \mu_{1}+(m-x) \mu_{2}\right)\right] g(i, j, m)\right\}\right)\right\},
\end{aligned}
$$

where in each case $a^{+}$represents the positive part of $a$. These mappings represent the one-step cost associated with current workers, capacity increase/decrease decisions, and a terminal cost $g$. In order to construct the optimal infinite horizon value functions let $v_{0, \beta}=0$ and define the following sets of equations,

$$
\begin{aligned}
v_{n, \beta}(i, j, k) & =i h_{1}+j h_{2}+H_{\beta}^{P} v_{n-1, \beta}(i, j, k), \\
v_{\beta}(i, j, k) & =i h_{1}+j h_{2}+H_{\beta}^{P} v_{\beta}(i, j, k), \\
\rho+w(i, j, k) & =i h_{1}+j h_{2}+H_{1}^{P} w(i, j, k),
\end{aligned}
$$

where $P=U, F$, or $C$.

The system of equations (2.7) are referred to as the finite horizon optimality equations (FHOE), (2.8) the discounted cost optimality equations (DCOE) and (2.9) the average cost optimality equations (ACOE). It is well-known that a solution to (2.7) is such that $v_{n, \beta}$ is the optimal, $\beta$-discounted, $n$-stage cost for whichever problem is currently under consideration. Since the action set is finite, Proposition 1.7 of [7] implies that $v_{n, \beta} \rightarrow v_{\beta}$, a solution to the DCOE, as $n \rightarrow \infty$. Moreover, a policy that achieves the minimum in (2.7) ((2.8)) is $n$-stage cost (infinite horizon, discounted cost) optimal for the appropriate model. The average cost case is a bit more subtle since the recurrence structure of the Markov process induced by each policy must be considered. For the problems considered, when a solution $(w, \rho)$ of the ACOE (2.9) exists it is such that $\rho$ is the optimal cost (independent of the initial state) and $w(x)$, called a relative value function, is unique up to an additive constant.

The first result states that the finite and infinite horizon discounted cost value functions are non-decreasing in $i$ and $j$. In order to ease notation, let $\Delta_{i} g(i, j)=$ $g(i+1, j)-g(i, j)$ and $\Delta_{j} g(i, j)=g(i, j+1)-g(i, j)$. 
Lemma 2.1. $v_{n, \beta}^{P}$ and $v_{\beta}^{P}$ are non-decreasing in the number of customers in either queue for $P=U, C, F$.

Proof. We show the result for the unrestricted case. The other cases follow similarly. The $n=0$ case is trivial. Assume that it holds at $n-1$. Let $d_{n}(i+1, j)=\left(k^{\prime}, x^{\prime}\right)$ be the optimal number of workers and the number of workers to place at station 1 when in state $(i+1, j)$ at time $n$. If we use the same decision in state $(i, j)$ we have for $i \geq 1$

$$
\begin{aligned}
\Delta_{i} v_{n, \beta}^{U}(i, j) \geq & h_{1}+\beta\left(\lambda \Delta_{i} v_{n-1, \beta}^{U}(i+1, j)+x^{\prime} \mu_{1} \Delta_{i} v_{n-1, \beta}^{U}(i-1, j+1)\right. \\
& \left.+\left(k^{\prime}-x^{\prime}\right) \mu_{2} \Delta_{i} v_{n, \beta}^{U}(i, j-1)+\left[1-\left(\lambda+x^{\prime} \mu_{1}+\left(k^{\prime}-x^{\prime}\right) \mu_{2}\right)\right] \Delta_{i} v_{n, \beta}^{U}(i, j)\right) .
\end{aligned}
$$

The inductive hypothesis and the non-negativity of $h_{1}$ implies $\Delta_{i} v_{n, \beta}^{U}(i, j) \geq 0$. A similar argument holds for $i=0$ except that we idle the workers assigned to station 1 in the process starting in state $(0, j)$. Taking limits as $n \rightarrow \infty$ yields the result in the infinite horizon case.

The next result yields that the ACOE holds in states that are positive recurrent for the average optimal policy. In Section 3 we provide results that allow us to identify the set of positive recurrent states.

Proposition 2.2. Suppose that in the unrestricted or controlled capacity reduction models there exists $k^{*} \in\{0,1, \ldots, \ell\}$ such that $\frac{\lambda}{\mu_{1}}+\frac{\lambda}{\mu_{2}}<k^{*}$ or in the uncontrolled capacity reduction case $\frac{\lambda}{\mu_{1}}+\frac{\lambda}{\mu_{2}}<z \equiv \sum_{k=1}^{\ell}\left(k p_{k}\right)$, where

$$
\begin{aligned}
& p_{0}=\left(1+\sum_{k=1}^{\ell} \frac{\Pi_{n=0}^{k-1} \alpha(n)}{k ! \gamma^{k}}\right)^{-1}, \\
& p_{k}=\frac{\prod_{n=0}^{k-1} \alpha(n)}{k ! \gamma^{k}} p_{0}, \quad k=1,2, \ldots, \ell .
\end{aligned}
$$

In each case, the following hold

1. The optimal average cost may be computed by $\rho=\lim _{\beta \uparrow 1}(1-\beta) v_{\beta}(x)$ for any $x \in \mathbb{X}$.

2. There exists a (bounded) limit point $w(x)=\lim _{n \rightarrow \infty} w_{\beta_{n}}(x)=\lim _{n \rightarrow \infty}\left[v_{\beta_{n}}(x)-\right.$ $v_{\beta_{n}}(\mathbf{0})$ ] such that $(\rho, w)$ satisfy the ACOE with the equality replaced with " $\geq$ ", where $v_{\beta}(\mathbf{0})=v_{\beta}^{U}(0,0)$ in the unrestricted case and $v_{\beta}(\mathbf{0})=\min _{k \in\{0,1, \ldots, \ell\}}\left\{v_{\beta}^{P}(0,0, k)\right\}$ for $P=C, F$.

3. Any policy, say $f$, achieving the minimum on the right hand side of the above inequality is average cost optimal with average cost $\rho$. Moreover, the inequality is an equality at any state that is positive recurrent under the Markov chain induced by $f$.

Proof. Lemma 2.1 yields that $v_{\beta}$ is non-decreasing in the number of customers in each queue. Moreover, under the hypotheses of the proposition we have the existence of a 
policy with finite average cost (see the Appendix) so that the assumptions of Sennott [24, Proposition 7.2.4] hold. The result now follows by applying Theorems 7.2.3 and 7.4.3 of [24].

The remainder of the paper is dedicated to proving Theorem 2.3 below. The results on the allocation decision hold for all three models, while the capacity decisions are for the unrestricted model. In the latter, the monotonicity results serve as a baseline for heuristics in the controlled and uncontrolled capacity decrease models.

Theorem 2.3. In each scenario and under the infinite horizon discounted cost and average cost criteria, the following hold

1. there exists an optimal policy that allocates all workers to one station or the other (workers are not split between the two)

2. if $\mu_{2} h_{2} \leq(\geq) \mu_{1}\left(h_{1}-h_{2}\right)$, there exists an optimal policy that is exhaustive in station $1(2)$.

Moreover, in the unrestricted case, if $\mu_{1}=\mu_{2}$,

3. for each fixed $(i, j) \in \mathbb{X}^{U}$ there exists an optimal number of workers, $L(i, j)$, such that $L(i, j)$ is non-decreasing in $i$ and $j$.

The third result above requires more comment. In all three cases, all of the examples we considered yielded that the optimal capacity increase/decrease policy was non-decreasing in $i$ and $j$. However, we were only able to prove it in the unrestricted case. The difficulty is that the optimal capacity increase/decrease decisions in the other cases may vary in three directions $(i, j, k)$. This significantly complicates the problem. Indeed, given the results for the unrestricted case, we originally conjectured that an optimal policy in the controlled and uncontrolled cases would continue to increase workers until reaching some level, say $M(i, j)$, after which it would stop increasing; monotone in $k$. The next example disproves this conjecture.

Example 2.4. Suppose we have the following inputs for the uncontrolled capacity reduction model: $h_{1}=2 ; h_{2}=3 ; \ell=7 ; \lambda=3 ; \mu_{1}=2 ; \mu_{2}=2 ; \alpha(k)=2 ; \gamma=.001$. Figure 1(a) displays the cost function $r(k)$ and 1(b) the average optimal capacity increase actions for $j=0,1$.

The second result of Theorem 2.3 implies that the optimal allocation is exhaustive in queue 2 so that the set of recurrent states may only have $j=0$ or 1 . Note that despite the fact that the cost function is non-decreasing in $k$, the optimal capacity increase policy is not. We note that the cost function depicted in Figure 1(a) may be interpreted as a system where the first few workers allocated are not costly while allocating several workers to this station significantly hampers productivity of the whole system.

We also mention that the inequality $\mu_{2} h_{2} \geq(\leq) \mu_{1}\left(h_{1}-h_{2}\right)$ is analogous to the $c \mu$-rule in parallel systems. In a parallel system, the choice of which job to serve next 


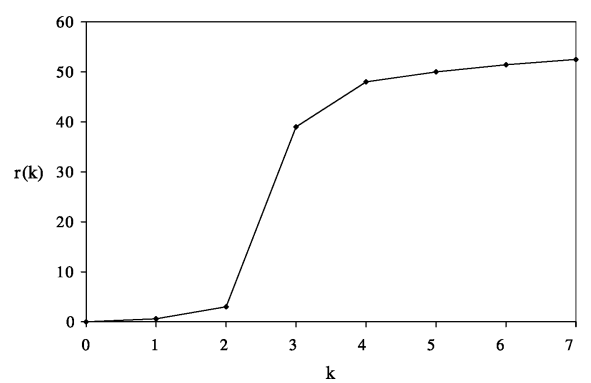

(a) Worker cost function.

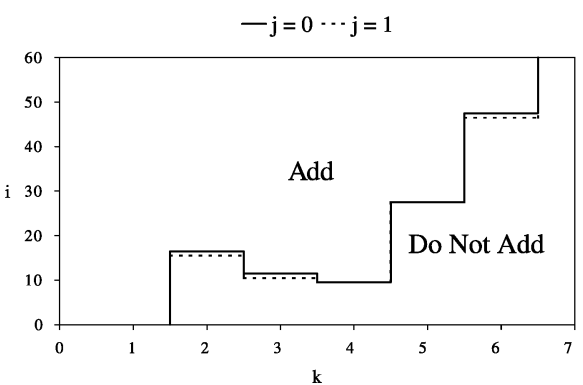

(b) Uncontrolled reduction optimal capacity increase policy.

Figure 1. Counter-example, Example 2.4, showing an optimal policy need not be monotone (in $k$ ).

is made based on the one that can reduce the cost the fastest; with the highest $c \mu$. The fact that the system has flexible workers makes the decision of where to serve a direct analogue. The right (left) hand side of the inequality is the rate at which costs can be drained if the workers are allocated to queue 1 (2). A further explanation is provided in [1].

\section{Optimal worker allocation}

We divide the control of the system into two parts: the first discusses how workers should be allocated to each station and the second discusses optimal capacity increase/decrease decisions. The next result shows that we need only consider policies that never divide the workers between queues. Although given the existence of a solution to the DCOE and ACOE, the proof is now simple, this result stands to simplify the other results of this section.

Proposition 3.1. In any of the models considered and under either the discounted or average cost criterion, there exists an optimal policy that always allocates all workers to either station 1 or station 2.

Proof. Note that the portion of $H_{\beta}^{U} g, H_{\beta}^{F} g$ and $H_{\beta}^{C} g$ that corresponds to the allocation decision has the form

$$
\begin{aligned}
G(i, j, k, x) \equiv & x \mu_{1} g(i-1, j+1, k)+(k-x) \mu_{2} g(i, j-1, k) \\
& +\left[1-\left(\lambda+k \gamma+\alpha(k)+x \mu_{1}+(k-x) \mu_{2}\right)\right] g(i, j, k),
\end{aligned}
$$

with perhaps $\gamma$ and $\alpha(k)$ being zero. Since $G(i, j, k, x)$ is linear in $x$ and a linear function achieves its maximum and minimum at the extreme points, the fact that the optimality equations under each criterion have a solution imply that it is optimal to set $x$ equal to 0 or $k$ and the result is proven. 
Proposition 3.1 implies that we may restrict attention to policies that always allocate all workers to one station or the other. Define $\Pi^{R} \subseteq \Pi$ to be this set of policies. Next we show the existence of optimal policies that are exhaustive in either queue 1 or 2 . The proof is analogous to that in Nain [23] for scheduling in parallel queues. First we make the assumption that the cost rate of having workers in the system is zero $(r(\cdot)=0)$. This will be relaxed later. For a policy $f$ let

$$
\phi^{f}(s) \equiv \int_{0}^{s} Z^{f}(x-)\left[\mu_{1}\left(h_{1}-h_{2}\right) a_{1}^{f}(x)+\mu_{2} h_{2} a_{2}^{f}(x)\right] d x
$$

where $Z^{f}(x)$ is the controlled Markov process representing the number of workers currently available, $a_{1}^{f}(x) \equiv 1_{\left\{Q_{1}^{f}(x-)>0, d^{f}(x)=1\right\}}, a_{2}^{f}(x) \equiv 1_{\left\{Q_{2}^{f}(x-)>0, d^{f}(x)=2\right\}}$, and $d^{f}(x)=i$ represents the action that all workers are currently assigned to station $i$, for $i=1,2$.

Lemma 3.2. For a fixed policy $f \in \Pi^{R}$ and fixed $\theta \geq 0$,

$v_{t, \theta}^{f}=\frac{1}{\theta}\left(1-e^{-\theta t}\right) \mathbb{E}\left[h_{1} Q_{1}^{f}(0)+h_{2} Q_{2}^{f}(0)\right]+\mathbb{E} \int_{0}^{t} e^{-\theta s} h_{1} A_{1}(s) d s-\mathbb{E} \int_{0}^{t} e^{-\theta s} \phi_{f}(s) d s$,

where $A_{1}(s)$ is a Poisson process of rate $\lambda$.

Proof. Denote the history of possible arrivals, services and the Markov process generated by the capacity increase/decrease decisions under policy $f$ by

$$
H(t)=\left\{\left(A_{1}(s), Y_{k}^{m}(s), Z^{f}(s)\right), k=1,2, \ldots, \ell, 0 \leq s \leq t\right\},
$$

where $\left\{Y_{k}^{m}, k=1,2, \ldots, \ell\right\}$ are independent Poisson processes of rate $\mu_{m}$ for $m=1,2$. Let $\mathcal{F}_{t}$ be the $\sigma$-field generated by $H(t)$. That is to say that $\mathcal{F}_{t}$ is the smallest filtration to which $H(t)$ is adapted. The queue length processes can be written

$$
\begin{aligned}
& Q_{1}^{f}(s)=Q_{1}^{f}(0)+A_{1}(s)-\sum_{k=1}^{\ell} \int_{0}^{s} 1_{\left\{Z^{f}(x-) \geq k\right\}} a_{1}^{f}(x) d Y_{k}^{1}(x) \\
& Q_{2}^{f}(s)=Q_{2}^{f}(0)+\sum_{k=1}^{\ell} \int_{0}^{s} 1_{\left\{Z^{f}(x-) \geq k\right\}} a_{1}^{f}(x) d Y_{k}^{1}(x)-\sum_{k=1}^{\ell} \int_{0}^{s} 1_{\left\{Z^{f}(x-) \geq k\right\}} a_{2}^{f}(x) d Y_{k}^{2}(x) .
\end{aligned}
$$

Note that since $f$ is non-anticipating (and thus, left-continuous) the integrands in (3.2) and (3.3) are predictable with the respect to $\mathcal{F}_{t}$. Moreover, each $Y_{k}^{m}(t)$ is adapted to 
$\mathcal{F}_{t}$. Applying a result from Bremaud [8] (see the "Partial result" on page 24 of [8]) we have

$$
\begin{aligned}
\mathbb{E} \sum_{k=1}^{\ell} \int_{0}^{s} 1_{\left\{Z^{f}(x-) \geq k\right\}} a_{m}^{f}(x) d Y_{k}^{m}(x) & =\mathbb{E} \sum_{k=1}^{\ell} \int_{0}^{s} 1_{\left\{Z^{f}(x-) \geq k\right\}} a_{m}^{f}(x) \mu_{m} d x \\
& =\mathbb{E} \int_{0}^{s} Z^{f}(x-) a_{m}^{f}(x) \mu_{m} d x,
\end{aligned}
$$

for $m=1,2$. Thus,

$$
\begin{aligned}
v_{t, \theta}^{f}= & \mathbb{E} \int_{0}^{t} e^{-\theta s} h_{1}\left[Q_{1}^{f}(0)+A_{1}(s)-\int_{0}^{s} Z^{f}(x-) a_{1}^{f}(x) \mu_{1} d x\right] d s \\
& +\mathbb{E} \int_{0}^{t} e^{-\theta s} h_{2}\left[Q_{2}^{f}(0)+\int_{0}^{s} Z^{f}(x-) a_{1}^{f}(x) \mu_{1} d x-\int_{0}^{s} Z^{f}(x-) a_{2}^{f}(x) \mu_{2} d x\right] d s .
\end{aligned}
$$

A little algebra yields

$v_{t, \theta}^{f}=\frac{1}{\theta}\left(1-e^{-\theta t}\right) \mathbb{E}\left[h_{1} Q_{1}^{f}(0)+h_{2} Q_{2}^{f}(0)\right]+\mathbb{E} \int_{0}^{t} e^{-\theta s} h_{1} A_{1}(s) d s-\mathbb{E} \int_{0}^{t} e^{-\theta s} \phi_{f}(s) d s$,

and the result is proven.

Note that the above proof does not require the Poisson arrival or worker availability process assumptions; any counting processes will suffice. Moreover, since only the last term in (3.1) is dependent on the policy, showing that $\pi^{*}$ minimizes $v_{t, \theta}^{\pi}$ is reduced to showing $\mathbb{E} \phi_{\pi^{*}}(s) \geq \mathbb{E} \phi_{\pi}(s)$ for all $\pi \in \Pi^{R}$ and all $0 \leq s \leq t$.

Theorem 3.3. Fix $t \geq 0$. Suppose $\mu_{1}\left(h_{1}-h_{2}\right) \leq(\geq) \mu_{2} h_{2}$. Then there exists a policy that minimizes $v_{s, \theta}^{\pi}$ over $\Pi^{R}$ for all $0 \leq s \leq t$ that allocates all available workers to queue $2(1)$ at time $x$ if $Q_{2}^{\pi}(x)>0\left(Q_{1}^{\pi}(x)>0\right)$.

Proof. Consider the case with $\mu_{1}\left(h_{1}-h_{2}\right) \leq \mu_{2} h_{2}$. The other case is proved similarly and is omitted for brevity. The result is proved via an interchange argument. Consider 2 processes defined on the same probability space. Suppose Process 1 follows a policy $f \in \Pi^{R}$ while Process 2 follows a policy $\pi \in \Pi^{R}$ defined shortly. Since they are defined on the same space, the arrivals and (potential) services are the same. Moreover, assume that $\pi$ is chosen so that the Markov chains $Z^{f}(s)$ and $Z^{\pi}(s)$ are such that $Z^{f}(s)=Z^{\pi}(s)$ almost surely. Suppose Process 1 allocates all workers to station 1 at some time $\sigma<t$ when $Q_{2}^{f}(\sigma)>0$. Let $\tau$ be the first time after $\sigma$ that Process 1 begins serving at station 2 and $\eta_{1}$ be the time after $\sigma$ that it completes service of the first customer at station 2. Note that since customers may be pre-empted, there is no reason to believe that $\tau$ corresponds to a service completion. 
The policy $\pi$ follows the same policy as $f$ until time $\sigma$. At this time, it serves at station 2 until either it completes service of the first customer in queue, or Process 1 stops serving at station 1; whichever occurs first. Denote this time by $\sigma^{*}$. Process 2 then follows the policy $f$ until such time that the first customer in queue 2 has been served (in Process 2). Denote this time by $\eta_{2}$. On $\left[\eta_{2}, \eta_{1}\right.$ ) Process 2 serves at station 1 . After $\eta_{1}$ the processes follow the same policy, $f$. Since the processes have the same number of workers, the amount of work that can be done before $\eta_{1}$ is the same. Moreover, since each process does precisely enough work at station 2 to serve the first customer (the rest of the capacity is spent serving station 1), after $\eta_{1}$ the two processes coincide. Thus, $Q_{k}^{f}(x)=Q_{k}^{\pi}(x)$ for $x \in[0, \sigma) \cup\left[\eta_{1}, \infty\right)$. Note that

$$
\begin{aligned}
& a_{1}^{\pi}(x)-a_{1}^{f}(x)= \begin{cases}0 & \text { if } x \in[0, \infty) \backslash\left(\left[\sigma, \sigma^{*}\right) \cup\left[\eta_{2}, \eta_{1}\right)\right), \\
-1 & \text { if } x \in\left[\sigma, \sigma^{*}\right), \\
1 & \text { if } x \in\left[\eta_{2}, \eta_{1}\right),\end{cases} \\
& a_{2}^{\pi}(x)-a_{2}^{f}(x)= \begin{cases}0 & \text { if } x \in[0, \infty) \backslash\left(\left[\sigma, \sigma^{*}\right) \cup\left[\eta_{2}, \eta_{1}\right)\right), \\
1 & \text { if } x \in\left[\sigma, \sigma^{*}\right), \\
-1 & \text { if } x \in\left[\eta_{2}, \eta_{1}\right) .\end{cases}
\end{aligned}
$$

Consider

$$
\begin{aligned}
\phi_{\pi}(s)-\phi_{f}(s) & =\int_{0}^{s} Z^{f}(x) \mu_{1}\left(h_{1}-h_{2}\right)\left(a_{1}^{\pi}(x)-a_{1}^{f}(x)\right)+\mu_{2} h_{2}\left(a_{2}^{\pi}(x)-a_{2}^{f}(x)\right) d x \\
& =\left[\mu_{2} h_{2}-\mu_{1}\left(h_{1}-h_{2}\right)\right] \int_{0}^{s} Z^{f}(x)\left(1_{\left\{x \in\left[\sigma, \sigma^{*}\right)\right\}}-1_{\left\{x \in\left[\eta_{2}, \eta_{1}\right)\right\}}\right) d x .
\end{aligned}
$$

Note that since the total amount of work done by each process must coincide, for $s \geq \eta_{1}$ the above integral is zero. On the other hand, since $\left[\sigma, \sigma^{*}\right) \cap\left[\eta_{2}, \eta_{1}\right)=\emptyset$, and $\sigma^{*} \leq \eta_{2}$, for $s<\eta_{1}$ the above integral is non-negative. Now, the assumption $\mu_{2} h_{2} \geq \mu_{1}\left(h_{1}-h_{2}\right)$ implies $\phi_{\pi}(s) \geq \phi_{f}(s)$ for each $0 \leq s \leq t$.

It should be clear that the above argument can be repeated to create a finite sequence of policies $\left\{\pi_{i}, 0 \leq i \leq M\right\}$ such that $\pi_{M}$ is exhaustive in station 2 and $\phi_{\pi_{i+1}}(s) \geq \phi_{\pi_{i}}(s)$ for each $0 \leq s \leq t$ where $\pi_{0}=f$.

As in [23], we note that although the policies $\pi_{i}$, for $1 \leq i \leq M-1$, are anticipating and thus not in $\Pi, \pi_{M}$ is non-anticipating (since it is exhaustive). Moreover, since $\pi_{M}$ only depends on $f$ through the capacity increase/decrease decisions, the increase/decrease process does not violate the non-anticipation. The optimal policy's capacity increase/decrease policy may improve on this by using the queue length information currently available. That is, there exists an exhaustive policy that has expected cost lower than or equal to that of $\pi_{M}$ and the result is proven.

Note that since the policy $\pi$ constructed in the proof of Theorem 3.3 has the same capacity increase/decrease process as that of $f$, the restriction that $r=0$ is not necessary. 
Moreover, we note that since the results of Theorem 3.3 hold for each $t$, they hold under the infinite horizon discounted and average cost cases (assuming stability) as well. In light of Proposition 3.1 they hold over the larger class of policies $\Pi$.

The results of this section suggest that the optimal capacity increase/decrease decisions are in some sense decoupled from the allocation decision. Originally we expected the two decisions to be intertwined, however, since the $c \mu$-rule is basically a "greedy" algorithm (drain the cost as fast as possible), this interpretation continues to hold no matter how much capacity is available. In the next section we analyze how capacity increase/decrease decisions are made.

\section{Optimal capacity increase/decrease}

In this section we consider the problem of when to increase and decrease workers. In the unrestricted model we show that the number of workers that should be made available is non-decreasing in both queue lengths. Although this result has also held true in all of our numerical studies in the controlled and uncontrolled capacity reduction cases, we have been unable to prove it to date. Recall from Example 2.4 that this monotonicity does not extend to the optimal number of workers.

Theorem 3.3 implies that when $\mu_{2} h_{2} \geq(\leq) \mu_{1}\left(h_{1}-h_{2}\right)$ we may restrict attention to policies that are exhaustive in queue 2 (1). Consider the unrestricted case and suppose $\mu_{2} h_{2} \geq \mu_{1}\left(h_{1}-h_{2}\right)$. For a (finite) function $g$ on $\mathbb{X}^{U}, H_{\beta}^{U}$ can now be simplified

$$
H_{\beta}^{U} g(i, j)= \begin{cases}\min _{k \in\{0,1, \ldots, \ell\}}\left\{r(k)+\beta\left(\lambda g(i+1, j)+k \mu_{2} g(i, j-1)\right.\right. & \\ \left.\left.\quad+\left[1-\left(\lambda+k \mu_{2}\right)\right] g(i, j)\right)\right\} & \\ \min _{k \in\{0,1, \ldots, \ell\}}\left\{r(k)+\beta\left(\lambda g(i+1, j)+k \mu_{1} g(i-1, j+1)\right.\right. & \\ \left.\left.\quad+\left[1-\left(\lambda+k \mu_{1}\right)\right] g(i, j)\right)\right\} & \text { for } j \geq 1,\end{cases}
$$

where for $i=j=0$ set $k=0$ in the minimum above. There is an analogous mapping with $k \mu_{2} g(i, j-1)$ replaced with $k \mu_{1} g(i-1, j+1)$ for the case with $\mu_{2} h_{2}<\mu_{1}\left(h_{1}-h_{2}\right)$.

Inspecting $H_{\beta}^{U} g(i, j)$, we note that if $r(k)$ is concave, the minimum is that of a concave function. Thus, its minimum is achieved on the boundary (i.e. at $k=0$ or $\ell$ ). This is stated in the following proposition.

Proposition 4.1. If $r(k)$ is concave, it is optimal to have either zero workers or $\ell$ workers in the system.

When $r(k)$ is a general non-decreasing function but perhaps not concave, the optimal policy depends on the number of jobs in each queue as well as $r(k)$. Therefore, we investigate the structure of the optimal policy with respect to the number of jobs in the system. For the remainder of this section assume that $\mu \equiv \mu_{1}=\mu_{2}$ and that $r(k)$ is a general non-decreasing function. Since $\beta$ will be fixed throughout most of the section, we write $v_{n}$ for $v_{n, \beta}$. The main theorem of this section is stated next. 
Theorem 4.2. In the $n$-stage discounted cost problem, for each fixed $(i, j)$, there exists an optimal number of workers to be made available, say $L_{n, \beta}(i, j)$. Moreover, these levels are such that $L_{n, \beta}(i, j)$ is non-decreasing in both $i$ and $j$. Similarly for the infinite horizon discounted cost and, under the assumptions of Proposition 2.2, for average cost models.

Lemma 4.3. Consider the following cases.

1. If $2 h_{2} \geq h_{1}$, then for all $n \geq 0$,

(a) $\Delta_{i} v_{n}^{U}(i, j-1) \leq \Delta_{i} v_{n}^{U}(i, j)$ for $j \geq 1$,

(b) $\Delta_{j} v_{n}^{U}(i, j-1) \leq \Delta_{j} v_{n}^{U}(i, j)$, for $j \geq 1$,

(c) $\Delta_{i} v_{n}^{U}(i-1,1) \leq \Delta_{i} v_{n}^{U}(i, 0)$, for $i \geq 1$

(d) $\Delta_{j} v_{n}^{U}(i, 0) \geq v_{n}^{U}(i, 0)-v_{n}^{U}(i-1,1) \geq \Delta_{j} v_{n}^{U}(i-1,0)$.

2. If $h_{1}>2 h_{2}$, then for all $n \geq 0$,

(a) $\Delta_{j} v_{n}^{U}(i, j) \leq \Delta_{j} v_{n}^{U}(i, j+1)$ for $i, j \geq 0$,

(b) $\Delta_{j} v_{n}^{U}(i-1, j+1) \leq \Delta_{j} v_{n}^{U}(i, j)$ for $i \geq 1$ and $j \geq 0$.

Proof. See Appendix.

Proof of Theorem 4.2 (and 2.3 (3)). We consider the cases when the optimal allocation policy is exhaustive at station 1 and 2 separately.

Case 1. $h_{1} \leq 2 h_{2}$. Recall that this implies the optimal allocation is for all available workers to serve exhaustively at station 2 . Suppose instead of the optimal policy at stage $n$ we use $d_{n}(i, j)$ in state $(i+1, j)$ and $d_{n}(i+1, j)$ in state $(i, j)$. Then for $j \geq 1$

$$
\begin{aligned}
v_{n}^{U}(i, j)= & i h_{1}+j h_{2}+r\left(d_{n}(i, j)\right)+\lambda v_{n-1}^{U}(i+1, j)+d_{n}(i, j) \mu v_{n-1}^{U}(i, j-1) \\
+ & {\left[1-\left(\lambda+d_{n}(i, j) \mu\right)\right] v_{n-1}^{U}(i, j) } \\
\leq & i h_{1}+j h_{2}+r\left(d_{n}(i+1, j)\right)+\lambda v_{n-1}^{U}(i+1, j) \\
& +d_{n}(i+1, j) \mu v_{n-1}^{U}(i, j-1)+\left[1-\left(\lambda+d_{n}(i+1, j) \mu\right)\right] v_{n-1}^{U}(i, j),
\end{aligned}
$$

and

$$
\begin{aligned}
v_{n}^{U}(i+1, j)= & (i+1) h_{1}+j h_{2}+r\left(d_{n}(i+1, j)\right)+\lambda v_{n-1}^{U}(i+2, j) \\
+ & d_{n}(i+1, j) \mu v_{n-1}^{U}(i+1, j-1) \\
+ & {\left[1-\left(\lambda+d_{n}(i+1, j) \mu\right)\right] v_{n-1}^{U}(i+1, j) } \\
\leq & (i+1) h_{1}+j h_{2}+r\left(d_{n}(i, j)\right)+\lambda v_{n-1}^{U}(i+2, j) \\
& +d_{n}(i, j) \mu v_{n-1}^{U}(i+1, j-1)+\left[1-\left(\lambda+d_{n}(i, j) \mu\right)\right] v_{n-1}^{U}(i+1, j) .
\end{aligned}
$$


Thus,

$$
\begin{aligned}
\Delta_{i} v_{n}^{U}(i, j) \geq & h_{1}+\lambda \Delta_{i} v_{n-1}^{U}(i+1, j)+d_{n}(i+1, j) \mu \Delta_{i} v_{n-1}^{U}(i, j-1) \\
& +\left[1-\left(\lambda+d_{n}(i+1, j) \mu\right)\right] \Delta_{i} v_{n-1}^{U}(i, j),
\end{aligned}
$$

and

$$
\begin{aligned}
\Delta_{i} v_{n}^{U}(i, j) \leq & h_{1}+\lambda \Delta_{i} v_{n-1}^{U}(i+1, j)+d_{n}(i, j) \mu \Delta_{i} v_{n-1}^{U}(i, j-1) \\
+ & {\left[1-\left(\lambda+d_{n}(i, j) \mu\right)\right] \Delta_{i} v_{n-1}^{U}(i, j) . }
\end{aligned}
$$

Subtracting (4.1) from (4.2) (and dividing by $\mu$ ) yields

$$
\left[d_{n}(i, j)-d_{n}(i+1, j)\right]\left(\Delta_{i} v_{n-1}^{U}(i, j-1)-\Delta_{i} v_{n-1}^{U}(i, j)\right) \geq 0 .
$$

When $j=0$, and $i \geq 1$ a similar argument yields

$$
\left[d_{n}(i, 0)-d_{n}(i+1,0)\right]\left(\Delta_{i} v_{n-1}^{U}(i-1,1)-\Delta_{i} v_{n-1}^{U}(i, 0)\right) \geq 0 .
$$

By symmetry we have the following inequalities also hold for $j \geq 1$,

$$
\left[d_{n}(i, j)-d_{n}(i, j+1)\right]\left(\Delta_{j} v_{n-1}^{U}(i, j-1)-\Delta_{j} v_{n-1}^{U}(i, j)\right) \geq 0,
$$

and for $j=0$ and $i \geq 1$,

$$
\left[d_{n}(i, 0)-d_{n}(i, 1)\right]\left(v_{n-1}^{U}(i, 0)-v_{n-1}^{U}(i-1,1)-\Delta_{j} v_{n-1}^{U}(i, 0)\right) \geq 0 .
$$

As noted previously, when $i=j=0$ it should be clear that it is optimal to have zero workers and the monotonicity holds trivially. Lemma 4.3 implies that in (4.3)(4.6) we have the difference in the actions between two states multiplied by a nonpositive quantity. Consider for example (4.3). If $\Delta_{i} v_{n-1}^{U}(i, j-1)-\Delta_{i} v_{n-1}^{U}(i, j)<0$, $d_{n}(i, j)-d_{n}(i+1, j) \leq 0$ and the result is proven. We note that the minimum in $H_{\beta}^{U} v_{n-1}^{U}(i, j)$ is realized when $r(k)+k \mu\left[v_{n-1}^{U}(i, j-1)-v_{n-1}^{U}(i, j)\right]$ is minimized. Similarly, for $H_{\beta}^{U} v_{n-1}^{U}(i+1, j)$ and $r(k)+k \mu\left[v_{n-1}^{U}(i+1, j-1)-v_{n-1}^{U}(i+1, j)\right]$. Thus, if $\Delta_{i} v_{n-1}^{U}(i, j-1)-\Delta_{i} v_{n-1}^{U}(i, j)=0$ we may choose the number of workers in each state to be the same and the result is proven. The infinite horizon cases are precisely the same upon recalling that both $v_{\beta}^{U}$ and $w^{U}$, solutions to the DCOE and ACOE, respectively, can be obtained by taking limits. That is, the inequalities of Lemma 4.3 hold with $v_{n}^{U}$ replaced with either $v_{\beta}^{U}$ or $w^{U}$. This completes the proof of Case 1 .

Case 2. $h_{1}>2 h_{2}$. It is optimal to serve exhaustively at station 1 . We first show that $L_{n, \beta}(i, j)$ is non-decreasing in $j$. In an analogous manner to the case when $h_{1} \leq 2 h_{2}$, for $i \geq 1$

$$
\left[d_{n}(i, j)-d_{n}(i, j+1)\right]\left(\Delta_{j} v_{n-1}^{U}(i-1, j+1)-\Delta_{j} v_{n-1}^{U}(i, j)\right) \geq 0,
$$


and for $i=0$

$$
\left[d_{n}(0, j)-d_{n}(0, j+1)\right]\left(\Delta_{j} v_{n-1}^{U}(0, j-1)-\Delta_{j} v_{n-1}^{U}(0, j)\right) \geq 0 .
$$

The proof now follows in the same manner as Case 1 by applying the second set of inequalities in Lemma 4.3.

To prove the result for $i$, note that for $i \geq 1$

$$
\left[d_{n}(i, j)-d_{n}(i+1, j)\right]\left(\Delta_{i} v_{n-1}^{U}(i-1, j+1)-\Delta_{i} v_{n-1}^{U}(i, j)\right) \geq 0,
$$

and for $i=0$

$$
\left[d_{n}(0, j)-d_{n}(1, j)\right]\left(v_{n-1}^{U}(0, j+1)-v_{n-1}^{U}(0, j-1)-\Delta_{i} v_{n-1}^{U}(0, j)\right) \geq 0
$$

Thus, we must show that the following inequalities hold for all $n \geq 0$ :

1. $\Delta_{i} v_{n}^{U}(i-1, j+1)-\Delta_{i} v_{n}^{U}(i, j) \leq 0$ for $i \geq 0$,

2. $v_{n}^{U}(i, j+1)-v_{n}^{U}(i+1, j)-v_{n}^{U}(i, j-1)+v_{n}^{U}(i, j) \leq 0$ for $i \geq 0, j \geq 1$,

3. $v_{n}^{U}(i-1, j)-v_{n}^{U}(i, j-1) \leq 0$ for $i, j \geq 1$,

where the last inequality, required for the proof of the other two, is proven by choosing $d_{n}(i-1, j)$ in state $(i, j-1)$ and is omitted for brevity. For the first inequality, note that for $i \geq 2$

$$
\begin{aligned}
\Delta_{i} v_{n}^{U} & (i-1, j+1)-\Delta_{i} v_{n}^{U}(i, j) \\
\leq & \lambda\left[\Delta_{i} v_{n-1}^{U}(i, j+1)-\Delta_{i} v_{n-1}^{U}(i+1, j)\right] \\
& +d_{n}(i-1, j+1) \mu\left[\Delta_{i} v_{n}^{U}(i-2, j+2)-\Delta_{i} v_{n-1}^{U}(i-1, j+1)\right] \\
& +\left[1-\left(\lambda+d_{n}(i+1, j) \mu\right)\right]\left[\Delta_{i} v_{n-1}^{U}(i-1, j+1)-\Delta_{i} v_{n-1}^{U}(i, j)\right],
\end{aligned}
$$

and for $i=1$

$$
\begin{aligned}
\Delta_{i} v_{n}^{U} & (0, j+1)-\Delta_{i} v_{n}^{U}(1, j) \\
\leq & \lambda\left[\Delta_{i} v_{n-1}^{U}(1, j+1)-\Delta_{i} v_{n-1}^{U}(2, j)\right] \\
& +d_{n}(0, j+1) \mu\left[v_{n-1}^{U}(0, j+2)-v_{n-1}^{U}(0, j)-\Delta_{i} v_{n-1}^{U}(0, j+1)\right] \\
& +\left[1-\left(\lambda+d_{n}(2, j) \mu\right)\right]\left[\Delta_{i} v_{n-1}^{U}(0, j+1)-\Delta_{i} v_{n-1}^{U}(1, j)\right] .
\end{aligned}
$$


The inductive hypothesis yields the result. For the second assertion with $i \geq 1$

$$
\begin{aligned}
& v_{n}^{U}(i, j+1)-v_{n}^{U}(i+1, j)-v_{n}^{U}(i, j-1)+v_{n}^{U}(i, j) \\
& \leq 2 h_{2}-h_{1}+\lambda\left[v_{n-1}^{U}(i+1, j+1)-v_{n-1}^{U}(i+2, j)-v_{n-1}^{U}(i+1, j-1)\right. \\
&\left.+v_{n-1}^{U}(i+1, j)\right]+\left[1-\left(\lambda+d_{n}(i+1, j) \mu\right)\right]\left[v_{n-1}^{U}(i, j+1)-v_{n-1}^{U}(i+1, j)\right. \\
&\left.-v_{n-1}^{U}(i, j-1)+v_{n-1}^{U}(i, j)\right]+d_{n}(i+1, j) \mu\left[v_{n-1}^{U}(i-1, j+2)-v_{n-1}^{U}(i, j+1)\right. \\
&\left.-v_{n-1}^{U}(i-1, j)+v_{n-1}^{U}(i-1, j+1)\right]+\left[d_{n}(i+1, j)-d_{n}(i, j-1)\right] \mu \\
& \times\left[v_{n-1}^{U}(i-1, j+2)-v_{n-1}^{U}(i, j+1)\right],
\end{aligned}
$$

and for $i=0$ and $j \geq 1$

$$
\begin{aligned}
& v_{n}^{U}(0, j+1)-v_{n}^{U}(1, j)-v_{n}^{U}(0, j-1)+v_{n}^{U}(0, j) \\
& \leq 2 h_{2}-h_{1}+\lambda\left[v_{n-1}^{U}(1, j+1)-v_{n-1}^{U}(2, j)-v_{n-1}^{U}(1, j-1)+v_{n-1}^{U}(1, j)\right] \\
&+\left[1-\left(\lambda+d_{n}(1, j) \mu\right)\right]\left[v_{n-1}^{U}(0, j+1)-v_{n-1}^{U}(1, j)-v_{n-1}^{U}(0, j-1)+v_{n-1}^{U}(0, j)\right] \\
&+d_{n}(0, j-1) \mu\left[\Delta_{j} v_{n-1}^{U}(0, j-2)-\Delta_{j} v_{n-1}^{U}(0, j)\right] .
\end{aligned}
$$

With the exception of the last term, in each case, the inductive hypothesis suffices for the non-positivity of each term. Furthermore, note that the inductive hypothesis implies, via (4.9) and (4.10), that $d_{n}(i+1, j) \geq d_{n}(i, j)$. Thus, since $d_{n}(i, j)$ is also non-decreasing in $j, d_{n}(i+1, j) \geq d_{n}(i, j-1)$. This completes the proof for $i \geq 1$. As for the last term for $i=0$, recall that $\Delta_{j} v_{n}^{U}(i+1, j) \geq \Delta_{j} v_{n}^{U}(i, j)$ so the result is proven.

As in the proof for Case 1, noting that solutions to the DCOE and ACOE can be obtained by taking limits, the results hold for the infinite horizon discounted and average cost cases.

\section{Numerical examples}

In this section we include examples under the average cost criterion and propose promising heuristics based on the results in Section 4. A truncated state space is used to facilitate computation. In the next two examples the optimal allocation is exhaustive in queue 2, and hence only states with $j=0$ or $j=1$ will be occupied in the long-run. Since $\mu_{1}=\mu_{2}$, the results of Theorem 2.3 part 3 also apply.

Example 5.1. Suppose we have the following parameter settings: $h_{1}=1 ; h_{2}=2$; $r(k)=k^{2} ; \ell=6 ; \lambda=.5 ; \mu_{1}=\mu_{2}=.5 ; \alpha(k)=.5 ; \gamma=.1$. Figure 2(a) displays the optimal capacity levels given the number of customers for the unrestricted model. Figure 2(b) displays the optimal capacity target levels for the controlled capacity reduction model; if $k$ is less than the target level, then it is optimal to add workers when the opportunities arise up to the target, and if $k$ is greater than the target level, then it is optimal to decrease the number of workers down to the target. For example, when $i=3$, 


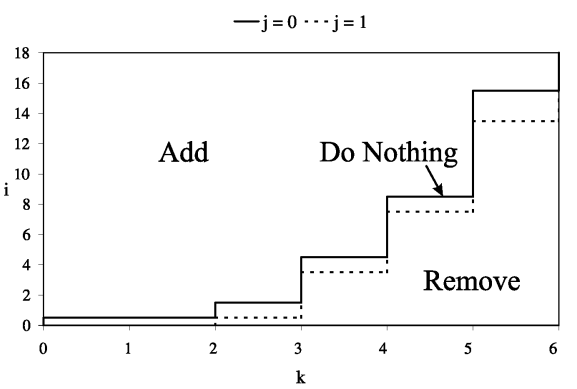

(a) Unrestricted optimal capacity levels.

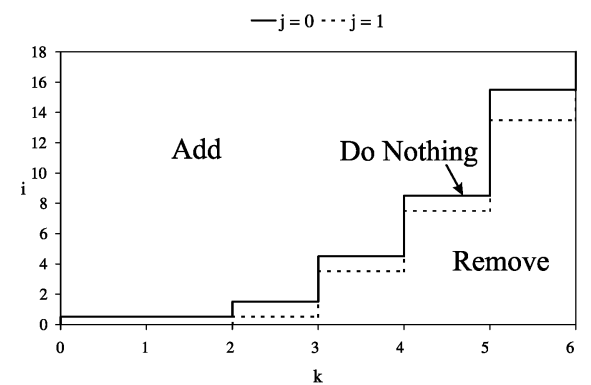

(b) Controlled reduction optimal capacity target levels: add (remove) workers when $k$ is less (more) than target.

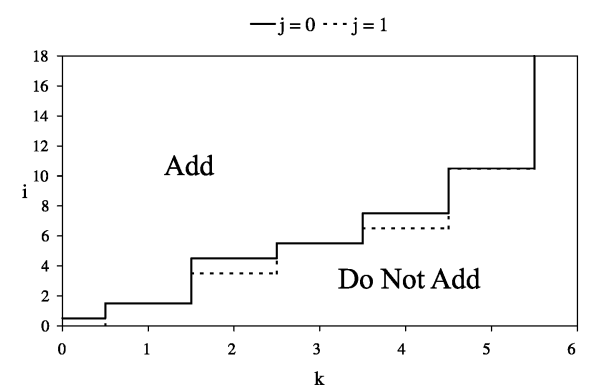

(c) Uncontrolled reduction optimal capacity increase policy.

Figure 2. Optimal policies for Example 5.1 with convex worker cost function $r(k)=k^{2}$.

$j=0$, it is optimal add workers if $k<3$, and remove workers when $k>3$. Figure 2(c) displays the optimal capacity increase policy for the uncontrolled reduction model. For example, when $i=3, j=0$, it is optimal to add workers if $k<2$.

The average cost in the unrestricted, controlled capacity reduction and uncontrolled capacity reduction cases is 7.6024, 8.2007 and 9.9530, respectively. As is expected, the unrestricted case dominates the controlled and uncontrolled capacity reduction cases (approximately $8 \%$ and $31 \%$, respectively). In the class of policies with a fixed number of servers, the optimal average cost is 11.0000 ( 3 workers is optimal in this class). This is approximately $45 \%$ more than the unrestricted case. This indicates (at least for this example) that a company can derive significant benefits from having dynamically flexible capacity.

We know from Theorem 2.3 part 3 that the optimal number of workers for the unrestricted case is monotone in the number of jobs. This is evident in Figure 2(a); the optimal capacity levels are increasing in both $i$ and $j$. The optimal policy for the controlled capacity reduction case, Figure 2(b), has a form close to the unrestricted policy and is also monotone in $i$ and $j$. In addition, it is monotone in $k$. Likewise, the optimal policy for the uncontrolled capacity reduction case, Figure 2(c), is monotone 
Table 1

Optimal average costs for Example 5.1 in the controlled reduction case for various $\alpha(k)=\alpha$. The second row compares this to the unrestricted optimal cost 7.6024.

\begin{tabular}{lcccccccccc}
\hline$\alpha$ & 0.1 & 0.2 & 0.5 & 1 & 2 & 5 & 10 & 20 & 50 & 100 \\
\hline Optimal cost & 8.8802 & 8.6651 & 8.2007 & 7.9933 & 7.9069 & 7.7243 & 7.6632 & 7.6328 & 7.6145 & 7.6085 \\
\% from unrestricted & $16.81 \%$ & $13.98 \%$ & $7.87 \%$ & $5.14 \%$ & $4.01 \%$ & $1.60 \%$ & $0.80 \%$ & $0.40 \%$ & $0.16 \%$ & $0.08 \%$ \\
\hline
\end{tabular}

Table 2

Uncontrolled reduction optimal average costs for Example 5.1 with various $\alpha(k)=\alpha$ and $\gamma$. NA indicates infeasibility. The minimum cost in each column is in bold.

\begin{tabular}{lcccccccccc}
\hline & \multicolumn{10}{c}{$\alpha$} \\
\cline { 2 - 11 }$\gamma$ & 0.1 & 0.2 & 0.5 & 1 & 2 & 5 & 10 & 20 & 50 & 100 \\
\hline 0.01 & $\mathbf{1 0 . 9 1 6 6}$ & $\mathbf{9 . 9 8 6 6}$ & 9.6393 & 9.5561 & 9.5159 & 9.4960 & 9.4874 & 9.4830 & 9.4804 & 9.4796 \\
0.05 & NA & 12.2387 & $\mathbf{9 . 3 9 8 2}$ & 8.9979 & 8.8438 & 8.7609 & 8.7337 & 8.7207 & 8.7133 & 8.7108 \\
0.1 & NA & NA & 9.9530 & $\mathbf{8 . 9 5 3 5}$ & $\mathbf{8 . 6 5 9 4}$ & 8.5054 & 8.4637 & 8.4449 & 8.4343 & 8.4309 \\
0.5 & NA & NA & NA & NA & 9.2114 & $\mathbf{8 . 2 9 8 0}$ & $\mathbf{8 . 0 9 5 1}$ & 8.0198 & 7.9797 & 7.9676 \\
1 & NA & NA & NA & NA & NA & 8.5664 & 8.1405 & $\mathbf{7 . 9 7 6 5}$ & $\mathbf{7 . 8 8 3 6}$ & 7.8555 \\
5 & NA & NA & NA & NA & NA & NA & NA & 8.5887 & 7.8991 & $\mathbf{7 . 7 7 3 7}$ \\
\hline
\end{tabular}

in all three state variables. As stated previously, while we were not able to prove it, we conjecture that the optimal policies for the controlled and uncontrolled reduction cases are monotone in $i, j$, and $k$ when $r(k)$ is non-decreasing and convex.

Tables 1 and 2 show the effect on the optimal average cost as $\alpha(k)$ is varied in the controlled reduction case, and $\alpha(k)$ and $\gamma$ are varied in the uncontrolled reduction case, respectively. In the controlled reduction case, as $\alpha(k)=\alpha$ becomes large, that is, when excess capacity becomes available more frequently, the average cost decreases towards that of the unrestricted case. For a fixed $\gamma$, the average cost also decreases in the uncontrolled reduction case as $\alpha$ increases. For a fixed $\alpha$, however, the average cost is not monotone in $\gamma$. For each $\alpha$ there is an "optimal" $\gamma$. If $\gamma$ is too low, excess workers stay too long, while if $\gamma$ is too high, the workers do not stay long enough. This suggests that heuristics based on the unrestricted case may do well in some cases and not well in others.

Example 5.2. Suppose we have the same parameters as in Example 5.1 except $r(k)=$ $15 \sqrt{k}$. Figure 3 displays the optimal capacity increase policy for the uncontrolled reduction model.

We have seen in Examples 2.4 and 5.2 that optimal policies need not be monotone in $k$ even when $r(k)$ is increasing. Our numerical studies suggest that such monotonicity is guaranteed only when $r(k)$ is convex. Example 5.2 has a worker cost function that is increasing and concave. We see in Figure 3 that the optimal policy for the uncontrolled 


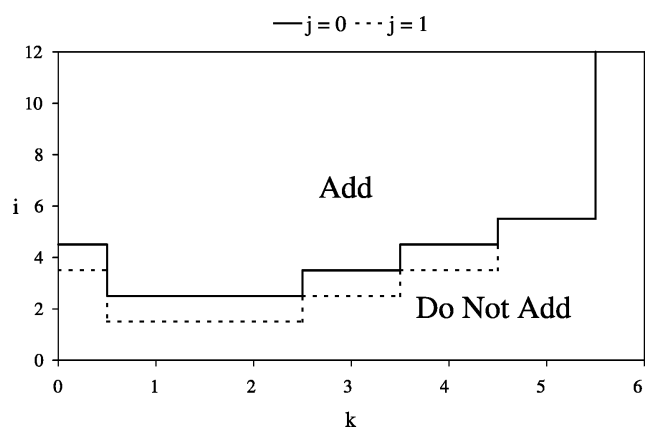

Figure 3. Uncontrolled reduction optimal capacity increase policy of Example 5.2 with concave worker cost function $r(k)=15 \sqrt{k}$.

reduction case is not monotone in $k$. For example, when $i=3$, it is optimal to not add workers when $k=0$, optimal to add workers when $k=1$, and then optimal to not add workers again for larger $k$.

\subsection{Heuristics}

In this section, we consider some heuristics that have shown promise. An easily characterized heuristic is to try to maintain a constant workforce level independent of the number of jobs in the system. As noted for Example 5.1, this does not work well in the unrestricted case (costs $45 \%$ more than the optimal policy). Instead consider the policy that increases capacity to a constant level, say $L$, when there are jobs in the system and then removes all workers when there are no jobs. We refer to such a policy as a $0-L$ heuristic. For the unrestricted case of Example 5.1, $L=3$ is optimal in this class of policies with an average cost of 8.0000 , only $5.23 \%$ above the optimal policy cost. For this system we exploited the fact that under a constant level of 3 workers, the probability of having zero customers in the system is significant: .3333. Thus, in this case, the $0-L$ heuristic significantly reduces the worker idle time.

For the controlled and uncontrolled reduction cases, a $0-L$ heuristic adds workers (when the opportunities arise) if and only if there are a positive number of jobs in the system and fewer than $L$ workers on hand. In addition in the controlled capacity reduction case, the heuristic policy removes all workers when there are no jobs in the system. The results for the controlled reduction case of Example 5.1 are given in Table 3 for various values of $\alpha(k)=\alpha$. Again $L=3$ performs the best. We see that for some parameters this heuristic performs well, while for other parameters it does not. In the uncontrolled capacity reduction case, the heuristic in general does not perform well. For example, for the parameters of Example 5.1 with $\alpha(k)=.5$ and $\gamma=.1$, the best such heuristic is only within $22 \%$ of optimal (average cost of 12.1440 compared to optimal 9.9530).

For the uncontrolled reduction case, consider the following policy: when there are less than $T$ jobs in the system (in total), add workers only when there are less than $L_{1}$ servers on hand; when there are $T$ or more jobs, add workers up to a total of 
Table 3

Average costs of a $0-L$ heuristic with $L=3$ applied to the controlled reduction model of Example 5.1 with various $\alpha(k)=\alpha$.

\begin{tabular}{lcccccccccc}
\hline$\alpha$ & 0.1 & 0.2 & 0.5 & 1 & 2 & 5 & 10 & 20 & 50 & 100 \\
\hline Heuristic cost & 14.3166 & 11.1361 & 9.1993 & 8.5555 & 8.0838 & 8.2476 & 8.0380 & 8.0179 & 8.0069 & 8.0034 \\
\% from optimal & $61.22 \%$ & $28.52 \%$ & $12.18 \%$ & $7.03 \%$ & $4.31 \%$ & $4.65 \%$ & $4.89 \%$ & $5.05 \%$ & $5.15 \%$ & $5.19 \%$ \\
\hline
\end{tabular}

$L_{2}$. Thus, there are two target worker levels, $L_{1}$ and $L_{2}$, and a threshold total number of jobs $T$ that indicates when to switch between the two. We refer to such a policy as a 2-level heuristic. A 2-level heuristic may perform well and is fairly simple to implement.

For Example 5.1, the best 2-level heuristic has $L_{1}=2, L_{2}=4$, and $T=3$. This is depicted in Figure 4(d). The average cost is 10.2438, within 2.92\% of optimal. Figure 4(b) shows a 2-level heuristic for Example 2.4 that performs within $0.47 \%$ of optimal. The best 2-level heuristic for Example 5.2 is depicted in Figure 4(f). Here, $L_{1}=0$ and $L_{2}=6$ and the policy reduces to a simple job threshold policy that adds capacity if and only if there are $T=4$ or more jobs in the system, independent of the number of workers (up to the capacity limit). This policy performs within $0.82 \%$ of optimal. It should be noted that a single job threshold policy $\left(L_{1}=0\right.$ and $\left.L_{2}=\ell\right)$ does not always perform well. For example, the best such policy for Example 5.1 has $T=3$ and is only within $17.63 \%$ of optimal.

\subsubsection{2-Level heuristic numerical study}

In this section we present the results of a numerical study of how well 2-level heuristics perform for a variety of parameters. For the study, the arrival rate and the total number of workers allowed are kept fixed at $\lambda=.5$ and $\ell=6$. Three values for the ratio $h_{2} / h_{1}$ are considered: .5, 2, and 5; $h_{1}=2$ and $h_{2}=1, h_{1}=1$ and $h_{2}=2$, and $h_{1}=1$ and $h_{2}=5$, respectively. There are also three possible values for the ratio $\mu_{1} / \mu_{2}: .5,1$, and 2 . The specific values for $\mu_{1}$ and $\mu_{2}$ are such that the offered load, $\lambda\left(1 / \mu_{1}+1 / \mu_{2}\right)$, is equal to 2 . The resulting values for $\mu_{1}$ and $\mu_{2}$ are $\mu_{1}=.5$ and $\mu_{2}=.5, \mu_{1}=.75$ and $\mu_{2}=.375$, and $\mu_{1}=.375$ and $\mu_{2}=.75$. Of the nine combinations of ratios $h_{2} / h_{1}$ and $\mu_{1} / \mu_{2}$, two combinations with $h_{2} / h_{1}=.5$ result in optimal exhaustive allocation at queue 1 (with one of these two being indifferent between allocation to queue 1 or to queue 2); the rest are exhaustive at queue 2.

For the uncontrolled reduction case, we consider three values for the ratio $\alpha / \gamma$. In order for the system to be stable, with an offered load of 2; it must be that $\alpha / \gamma>$ 2.0258. The ratios $\alpha / \gamma$ are chosen to be 3,5, and 10. As a base case, consider the convex worker cost function $r(k)=k^{2}$. For this cost function, $\gamma$ is chosen to be .05 , .1 , and .5. Hence, the base set of parameters have nine different $\alpha-\gamma$ combinations and 81 overall parameter combinations. In particular, the parameters of Example 5.1 are included. 


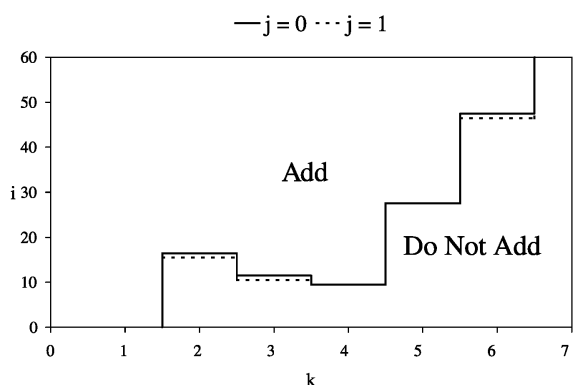

(a) Optimal capacity increase policy, Example 2.4.

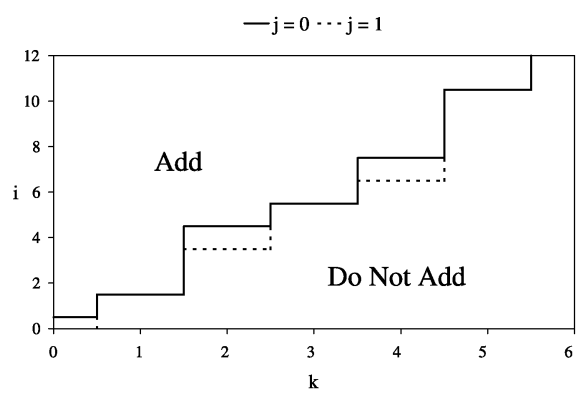

(c) Optimal capacity increase policy, Example 5.1.

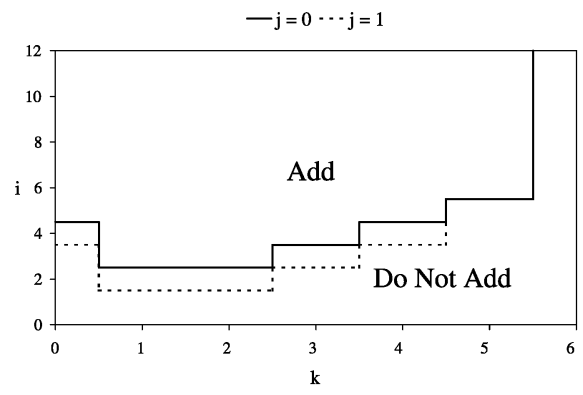

(e) Optimal capacity increase policy, Example 5.2.

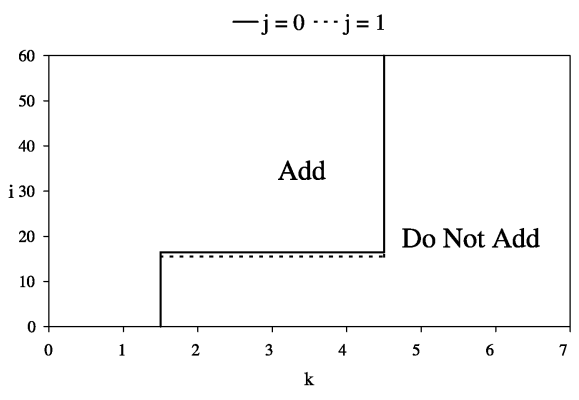

(b) Heuristic within $0.47 \%$ of optimal, Example 2.4

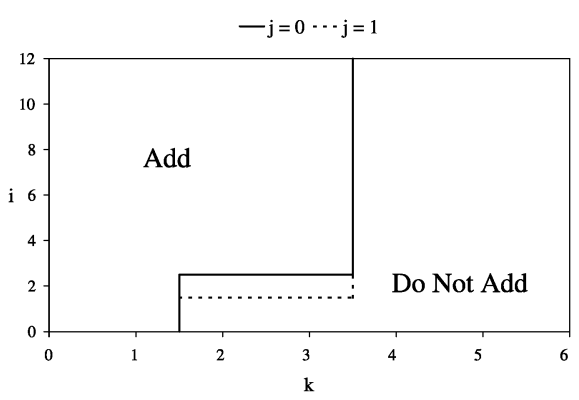

(d) Heuristic within $2.92 \%$ of optimal, Example 5.1 . $-\mathrm{j}=0 \cdots \mathrm{j}=1$

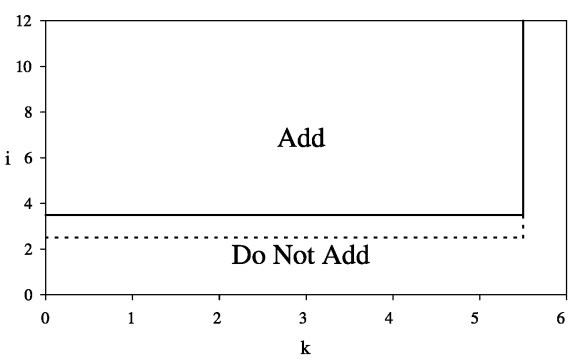

(f) Heuristic within $0.82 \%$ of optimal, Example 5.2

Figure 4. Optimal uncontrolled reduction capacity increase policies and respective heuristics.

The results of the numerical study are given in Table 4 . The average percent difference between the optimal average cost and the average cost of the best 2-level heuristic is $2.77 \%$. We see that the percent difference gets smaller as $\alpha$ decreases $(\alpha / \gamma$ decreases for a fixed $\gamma$ ). In fact, the average percent difference for $\alpha \leq \lambda=.5$ is only $1.77 \%$. When $\alpha / \gamma$ is 3 (or less), it turns out that the best 2-level heuristics always have $L_{2}=6$; full capacity. In these cases both the optimal policy and the heuristic policy spend a lot of time in states where it is optimal to hire, and the percent differences between their average costs are very small. As $\alpha$ increases, the percent of arrivals that are hired decreases. For the parameters of Example 5.1 with $\alpha=.5$, the best 2-level heuristic has $L_{1}=2, L_{2}=4$, 
Table 4

Results of the numerical study for the uncontrolled reduction base case with $r(k)=k^{2}$. Given are the optimal average cost, the average cost of the best 2-level heuristic, and the percent difference.

\begin{tabular}{|c|c|c|c|c|c|c|c|c|c|c|c|}
\hline & & & \multicolumn{3}{|c|}{$\frac{h_{2}}{h_{1}}=.5$} & \multicolumn{3}{|c|}{$\frac{h_{2}}{h_{1}}=2$} & \multicolumn{3}{|c|}{$\frac{h_{2}}{h_{1}}=5$} \\
\hline & & & $\frac{\mu_{1}}{\mu_{2}}=.5$ & $\frac{\mu_{1}}{\mu_{2}}=1$ & $\frac{\mu_{1}}{\mu_{2}}=2$ & $\frac{\mu_{1}}{\mu_{2}}=.5$ & $\frac{\mu_{1}}{\mu_{2}}=1$ & $\frac{\mu_{1}}{\mu_{2}}=2$ & $\frac{\mu_{1}}{\mu_{2}}=.5$ & $\frac{\mu_{1}}{\mu_{2}}=1$ & $\frac{\mu_{1}}{\mu_{2}}=2$ \\
\hline \multirow[t]{9}{*}{$\gamma=.05$} & \multirow[t]{3}{*}{$\frac{\alpha}{\gamma}=3$} & Opt: & 24.905 & 24.637 & 21.209 & 16.753 & 16.823 & 17.043 & 17.621 & 18.122 & 18.769 \\
\hline & & Heur: & 24.938 & 24.670 & 21.232 & 16.843 & 16.911 & 17.124 & 17.684 & 18.173 & 18.806 \\
\hline & & $\%$ Diff: & $0.14 \%$ & $0.13 \%$ & $0.11 \%$ & $0.54 \%$ & $0.52 \%$ & $0.48 \%$ & $0.36 \%$ & $0.28 \%$ & $0.20 \%$ \\
\hline & \multirow[t]{3}{*}{$\frac{\alpha}{\gamma}=5$} & Opt: & 13.805 & 13.594 & 12.452 & 10.786 & 10.881 & 11.074 & 11.632 & 12.138 & 12.750 \\
\hline & & Heur: & 13.953 & 13.737 & 12.606 & 11.064 & 11.143 & 11.333 & 11.871 & 12.355 & 12.947 \\
\hline & & $\%$ Diff: & $1.07 \%$ & $1.05 \%$ & $1.24 \%$ & $2.58 \%$ & $2.41 \%$ & $2.34 \%$ & $2.05 \%$ & $1.79 \%$ & $1.55 \%$ \\
\hline & \multirow{3}{*}{$\frac{\alpha}{\gamma}=10$} & Opt: & 11.344 & 11.148 & 10.393 & 9.312 & 9.398 & 9.593 & 10.140 & 10.652 & 11.248 \\
\hline & & Heur: & 11.732 & 11.529 & 10.794 & 9.630 & 9.698 & 9.907 & 10.462 & 10.947 & 11.572 \\
\hline & & $\%$ Diff: & $3.42 \%$ & $3.42 \%$ & $3.86 \%$ & $3.41 \%$ & $3.19 \%$ & $3.27 \%$ & $3.17 \%$ & $2.77 \%$ & $2.88 \%$ \\
\hline \multirow[t]{9}{*}{$\gamma=.1$} & \multirow[t]{3}{*}{$\frac{\alpha}{\gamma}=3$} & Opt: & 19.024 & 18.767 & 16.479 & 13.491 & 13.563 & 13.783 & 14.361 & 14.868 & 15.519 \\
\hline & & Heur: & 19.080 & 18.820 & 16.654 & 13.599 & 13.671 & 13.889 & 14.469 & 14.978 & 15.630 \\
\hline & & $\%$ Diff: & $0.30 \%$ & $0.28 \%$ & $1.06 \%$ & $0.80 \%$ & $0.80 \%$ & $0.77 \%$ & $0.76 \%$ & $0.74 \%$ & $0.72 \%$ \\
\hline & \multirow{3}{*}{$\frac{\alpha}{\gamma}=5$} & Opt: & 12.310 & 12.102 & 11.187 & 9.861 & 9.953 & 10.152 & 10.708 & 11.231 & 11.836 \\
\hline & & Heur: & 12.648 & 12.442 & 11.578 & 10.159 & 10.244 & 10.438 & 10.995 & 11.499 & 12.110 \\
\hline & & $\%$ Diff: & $2.74 \%$ & $2.81 \%$ & $3.50 \%$ & $3.03 \%$ & $2.92 \%$ & $2.82 \%$ & $2.68 \%$ & $2.39 \%$ & $2.31 \%$ \\
\hline & \multirow[t]{3}{*}{$\frac{\alpha}{\gamma}=10$} & Opt: & 10.663 & 10.471 & 9.813 & 8.848 & 8.954 & 9.160 & 9.696 & 10.202 & 10.793 \\
\hline & & Heur: & 11.044 & 10.850 & 10.166 & 9.166 & 9.261 & 9.451 & 10.020 & 10.516 & 11.106 \\
\hline & & $\%$ Diff: & $3.57 \%$ & $3.62 \%$ & $3.60 \%$ & $3.59 \%$ & $3.44 \%$ & $3.17 \%$ & $3.34 \%$ & $3.08 \%$ & $2.91 \%$ \\
\hline \multirow[t]{9}{*}{$\gamma=.5$} & \multirow[t]{3}{*}{$\frac{\alpha}{\gamma}=3$} & Opt: & 12.951 & 12.951 & 11.822 & 10.306 & 10.384 & 10.599 & 11.175 & 11.684 & 12.332 \\
\hline & & Heur: & 13.428 & 13.178 & 12.138 & 10.606 & 10.690 & 10.904 & 11.492 & 11.986 & 12.606 \\
\hline & & $\%$ Diff: & $3.69 \%$ & $1.76 \%$ & $2.67 \%$ & $2.91 \%$ & $2.94 \%$ & $2.87 \%$ & $2.84 \%$ & $2.58 \%$ & $2.23 \%$ \\
\hline & \multirow[t]{3}{*}{$\frac{\alpha}{\gamma}=5$} & Opt: & 10.325 & 10.140 & 9.578 & 8.712 & 8.825 & 9.007 & 9.548 & 10.045 & 10.619 \\
\hline & & Heur: & 10.870 & 10.666 & 10.081 & 9.083 & 9.168 & 9.366 & 9.932 & 10.443 & 11.064 \\
\hline & & \% Diff: & $5.28 \%$ & $5.19 \%$ & $5.25 \%$ & $4.26 \%$ & $3.89 \%$ & $3.98 \%$ & $4.02 \%$ & $3.96 \%$ & $4.19 \%$ \\
\hline & \multirow[t]{3}{*}{$\frac{\alpha}{\gamma}=10$} & Opt: & 9.491 & 9.358 & 8.893 & 8.190 & 8.298 & 8.498 & 9.042 & 9.524 & 10.072 \\
\hline & & Heur: & 10.121 & 9.921 & 9.429 & 8.659 & 8.753 & 8.941 & 9.504 & 9.999 & 10.571 \\
\hline & & \% Diff: & $6.64 \%$ & $6.02 \%$ & $6.02 \%$ & $5.73 \%$ & $5.49 \%$ & $5.20 \%$ & $5.11 \%$ & $4.98 \%$ & $4.96 \%$ \\
\hline
\end{tabular}

$T=3$, and a $2.92 \%$ percent difference; when $\alpha=.3$, the best heuristic is $L_{1}=3$, $L_{2}=6, T=4$, and the percent difference is only $0.80 \%$; when $\alpha=1$, the best heuristic is $L_{1}=2, L_{2}=4, T=5$, and the percent difference is $3.44 \%$. Increasing the opportunities to add workers makes the optimal policy in a sense more "dynamic" and the 2-level heuristic may not do as well in matching it. Below we will see results for other worker cost functions where sometimes the percent difference in costs may actually decrease as $\alpha$ increases.

So far we have only defined a 2-level heuristic for the uncontrolled reduction case. We can also define 2-level heuristics for the controlled reduction and unrestricted cases. In the unrestricted case, when there are less than $T$ jobs in system, have $L_{1}$ workers on 
Table 5

Results (optimal average cost, cost of the best 2-level heuristic, and percent difference) of the numerical study for the controlled reduction case of the base parameters with $r(k)=k^{2}$.

\begin{tabular}{|c|c|c|c|c|c|c|c|c|c|c|}
\hline & \multicolumn{3}{|c|}{$\frac{h_{2}}{h_{1}}=.5$} & \multicolumn{3}{|c|}{$\frac{h_{2}}{h_{1}}=2$} & \multicolumn{3}{|c|}{$\frac{h_{2}}{h_{1}}=5$} \\
\hline & & $\frac{\mu_{1}}{\mu_{2}}=.5$ & $\frac{\mu_{1}}{\mu_{2}}=1$ & $\frac{\mu_{1}}{\mu_{2}}=2$ & $\frac{\mu_{1}}{\mu_{2}}=.5$ & $\frac{\mu_{1}}{\mu_{2}}=1$ & $\frac{\mu_{1}}{\mu_{2}}=2$ & $\frac{\mu_{1}}{\mu_{2}}=.5$ & $\frac{\mu_{1}}{\mu_{2}}=1$ & $\frac{\mu_{1}}{\mu_{2}}=2$ \\
\hline \multirow[t]{3}{*}{$\alpha=.15$} & Opt: & 10.439 & 10.265 & 9.544 & 8.647 & 8.735 & 8.919 & 9.461 & 9.958 & 10.547 \\
\hline & Heur: & 10.620 & 10.418 & 9.672 & 8.737 & 8.819 & 9.012 & 9.560 & 10.054 & 10.651 \\
\hline & \% Diff: & $1.73 \%$ & $1.50 \%$ & $1.34 \%$ & $1.04 \%$ & $0.96 \%$ & $1.04 \%$ & $1.04 \%$ & $0.97 \%$ & $0.99 \%$ \\
\hline \multirow[t]{3}{*}{$\alpha=.25$} & Opt: & 10.131 & 9.948 & 9.336 & 8.530 & 8.626 & 8.806 & 9.337 & 9.822 & 10.398 \\
\hline & Heur: & 10.262 & 10.070 & 9.457 & 8.626 & 8.706 & 8.895 & 9.433 & 9.917 & 10.508 \\
\hline & \% Diff: & $1.30 \%$ & $1.22 \%$ & $1.30 \%$ & $1.13 \%$ & $0.92 \%$ & $1.01 \%$ & $1.03 \%$ & $0.97 \%$ & $1.06 \%$ \\
\hline \multirow[t]{3}{*}{$\alpha=.3$} & Opt: & 10.058 & 9.872 & 9.286 & 8.399 & 8.506 & 8.685 & 9.244 & 9.761 & 10.360 \\
\hline & Heur: & 10.172 & 9.983 & 9.399 & 8.601 & 8.680 & 8.868 & 9.402 & 9.883 & 10.470 \\
\hline & \% Diff: & $1.14 \%$ & $1.13 \%$ & $1.22 \%$ & $2.41 \%$ & $2.04 \%$ & $2.11 \%$ & $1.71 \%$ & $1.24 \%$ & $1.06 \%$ \\
\hline \multirow[t]{3}{*}{$\alpha=.5$} & Opt: & 9.768 & 9.591 & 8.974 & 8.104 & 8.201 & 8.373 & 8.909 & 9.404 & 9.980 \\
\hline & Heur: & 10.002 & 9.819 & 9.292 & 8.400 & 8.489 & 8.661 & 9.182 & 9.657 & 10.224 \\
\hline & \% Diff: & $2.40 \%$ & $2.38 \%$ & $3.53 \%$ & $3.65 \%$ & $3.51 \%$ & $3.43 \%$ & $3.06 \%$ & $2.69 \%$ & $2.44 \%$ \\
\hline \multirow[t]{3}{*}{$\alpha=1$} & Opt: & 9.266 & 9.099 & 8.606 & 7.903 & 7.993 & 8.160 & 8.670 & 9.140 & 9.691 \\
\hline & Heur: & 9.866 & 9.689 & 9.018 & 8.226 & 8.308 & 8.470 & 8.958 & 9.401 & 9.933 \\
\hline & \% Diff: & $6.48 \%$ & $6.49 \%$ & $4.79 \%$ & $4.09 \%$ & $3.94 \%$ & $3.80 \%$ & $3.32 \%$ & $2.86 \%$ & $2.50 \%$ \\
\hline \multirow[t]{3}{*}{$\alpha=1.5$} & Opt: & 9.106 & 8.942 & 8.486 & 7.846 & 7.934 & 8.098 & 8.597 & 9.056 & 9.597 \\
\hline & Heur: & 9.732 & 9.540 & 8.918 & 8.196 & 8.274 & 8.432 & 8.904 & 9.334 & 9.850 \\
\hline & \% Diff: & $6.87 \%$ & $6.68 \%$ & $5.09 \%$ & $4.46 \%$ & $4.30 \%$ & $4.13 \%$ & $3.58 \%$ & $3.07 \%$ & $2.63 \%$ \\
\hline \multirow[t]{3}{*}{$\alpha=2.5$} & Opt: & 8.985 & 8.822 & 8.392 & 7.761 & 7.847 & 8.005 & 8.486 & 8.931 & 9.452 \\
\hline & Heur: & 9.626 & 9.437 & 8.854 & 8.114 & 8.190 & 8.342 & 8.800 & 9.217 & 9.714 \\
\hline & \% Diff: & $7.14 \%$ & $6.97 \%$ & $5.52 \%$ & $4.54 \%$ & $4.38 \%$ & $4.22 \%$ & $3.70 \%$ & $3.20 \%$ & $2.77 \%$ \\
\hline \multirow[t]{3}{*}{$\alpha=5$} & Opt: & 8.792 & 8.635 & 8.221 & 7.639 & 7.724 & 7.882 & 8.359 & 8.802 & 9.312 \\
\hline & Heur: & 9.453 & 9.266 & 8.687 & 8.009 & 8.084 & 8.234 & 8.683 & 9.094 & 9.582 \\
\hline & \% Diff: & $7.51 \%$ & $7.31 \%$ & $5.67 \%$ & $4.84 \%$ & $4.65 \%$ & $4.46 \%$ & $3.88 \%$ & $3.32 \%$ & $2.89 \%$ \\
\hline
\end{tabular}

hand. When there are $T$ or more jobs, have $L_{2}$ workers. In the uncontrolled reduction case, hire when there are less than $T$ jobs and less than $L_{1}$ workers on hand. When there are less than $T$ jobs and more than $L_{1}$ workers, reduce capacity down to $L_{1}$. When there are $T$ or more jobs, hire up to or reduce down to $L_{2}$.

Results for 2-level heuristics in the controlled reduction and unrestricted cases for the base set of parameters are given in Tables 5 and 6, respectively. While there are nine different $\alpha / \gamma$ ratios in the uncontrolled reduction case, there are only eight different values for $\alpha$. These are the values studied in the controlled reduction case. The average difference in costs for the controlled reduction case (over 8 different $\alpha$ 's) is $3.16 \%$, with an average of only $1.71 \%$ for $\alpha \leq \lambda$. The average difference in costs for the unrestricted case is 5.32\%. For the parameters of Example 5.1, the best 2-level heuristic in the unrestricted case is $L_{1}=0, L_{2}=3$, and $T=1$ with a cost difference of $5.23 \%$. In other words, the best 2-level heuristic is a $0-L$ heuristic with $L=3$, the best $0-L$ heuristic. All of the best 2-level heuristics in the unrestricted case are $0-L$ heuristics. In 
Table 6

Results (optimal average cost, cost of the best 2-level heuristic, and percent difference) of the numerical study for the unrestricted case of the base parameters with $r(k)=k^{2}$.

\begin{tabular}{|c|c|c|c|c|c|c|c|c|c|}
\hline & \multicolumn{3}{|c|}{$\frac{h_{2}}{h_{1}}=.5$} & \multicolumn{3}{|c|}{$\frac{h_{2}}{h_{1}}=2$} & \multicolumn{3}{|c|}{$\frac{h_{2}}{h_{1}}=5$} \\
\hline & $\frac{\mu_{1}}{\mu_{2}}=.5$ & $\frac{\mu_{1}}{\mu_{2}}=1$ & $\frac{\mu_{1}}{\mu_{2}}=2$ & $\frac{\mu_{1}}{\mu_{2}}=.5$ & $\frac{\mu_{1}}{\mu_{2}}=1$ & $\frac{\mu_{1}}{\mu_{2}}=2$ & $\frac{\mu_{1}}{\mu_{2}}=.5$ & $\frac{\mu_{1}}{\mu_{2}}=1$ & $\frac{\mu_{1}}{\mu_{2}}=2$ \\
\hline Opt: & 8.544 & 8.387 & 8.007 & 7.517 & 7.602 & 7.760 & 8.233 & 8.671 & 9.170 \\
\hline Heur: & 9.185 & 9.000 & 8.476 & 7.926 & 8.000 & 8.148 & 8.593 & 9.000 & 9.481 \\
\hline \% Diff: & $7.50 \%$ & $7.31 \%$ & $5.87 \%$ & $5.43 \%$ & $5.23 \%$ & $5.00 \%$ & $4.36 \%$ & $3.79 \%$ & $3.40 \%$ \\
\hline
\end{tabular}

the uncontrolled case, the parameters of Example 5.1 result in a best 2-level heuristic of $L_{1}=1, L_{2}=3$, and $T=1$ with a cost difference of $3.51 \%$. This is better than the $12.18 \%$ of the best $0-L$ heuristic found in Table 3 . Note that in the controlled reduction case the cost difference is not always non-decreasing in $\alpha$.

In addition to the convex worker cost function $r(k)=k^{2}$ (Function 1), consider the linear function $r(k)=6 k$ (Function 2), the concave function in Example 5.2, $r(k)=15 \sqrt{k}$ (Function 3), and the general function (neither concave nor convex) given by the vector $r=[0,8,13,17,22,30,36]$ (Function 4). Numerical results for these functions are given in Tables 7-9 for the uncontrolled reduction, controlled reduction, and unrestricted cases, respectively. We only give results for the case $\gamma=.1$ and the three resulting $\alpha$ 's when $\alpha / \gamma=3,5$, and 10 .

For Function 1 the average cost difference in the uncontrolled case restricted to $\gamma=.1$ is $2.29 \%$. For Functions 2, 3, and 4 this average difference is $1.75 \%, 0.94 \%$, and $1.43 \%$, respectively. For Function 1 in the controlled firing case the overall average cost difference for $\alpha=.3, .5$, and 1 is $2.94 \%$. For Functions 2, 3, and 4 this average difference is $0.37 \%, 0.90 \%$, and $0.42 \%$, respectively. For Function 1 in the unrestricted case the average cost difference is 5.32\%. For Functions 2, 3, and 4 this average difference is $0.00 \%, 0.00 \%$, and $0.74 \%$. For Functions 2, 3, and 4 the 2-level heuristics do very well. In fact, for Functions 2 and 3, both of which are concave, the best 2-level heuristic in the unrestricted case is always $L_{1}=0, L_{2}=6$, and $T=1$, which coincides with the overall optimal policy. For Function 3, the best 2-level heuristic in the unrestricted case is always $L_{1}=0, L_{2}=4$, and $T=1$, a $0-L$ heuristic. Since it is not concave, it does not have $L_{2}=6$ and does not coincide exactly with the overall optimal policy. Note again that the percent difference in costs is not always non-decreasing in $\alpha$. For example, for the parameters of Example 5.1 except with worker cost Function 4, the best 2-level heuristic $\left(L_{1}=1, L_{2}=4, T=3\right)$ is within $1.98 \%$ of the optimal cost while the best 2-level heuristics for $\alpha$ lowered to $.3\left(L_{1}=3, L_{2}=6\right.$, $T=4)$ and $\alpha$ raised to $1\left(L_{1}=0, L_{2}=4, T=4\right)$ are within $0.53 \%$ and $1.53 \%$, respectively.

The 2-level heuristics displayed in Figures 4(b), (d), and (f) seem to work well because they are essentially smoothing the optimal policies, Figures 4(a), (c), and (e), respectively. While we found that 2 -level heuristics work well in many settings and are 
Table 7

Results (optimal average cost, cost of the best 2-level heuristic, and percent difference) of the numerical study for the uncontrolled reduction case of the base parameters restricted to $\gamma=.1$ with various worker cost functions.

\begin{tabular}{|c|c|c|c|c|c|c|c|c|c|c|c|}
\hline & & & \multicolumn{3}{|c|}{$\frac{h_{2}}{h_{1}}=.5$} & \multicolumn{3}{|c|}{$\frac{h_{2}}{h_{1}}=2$} & \multicolumn{3}{|c|}{$\frac{h_{2}}{h_{1}}=5$} \\
\hline & & & $\frac{\mu_{1}}{\mu_{2}}=.5$ & $\frac{\mu_{1}}{\mu_{2}}=1$ & $\frac{\mu_{1}}{\mu_{2}}=2$ & $\frac{\mu_{1}}{\mu_{2}}=.5$ & $\frac{\mu_{1}}{\mu_{2}}=1$ & $\frac{\mu_{1}}{\mu_{2}}=2$ & $\frac{\mu_{1}}{\mu_{2}}=.5$ & $\frac{\mu_{1}}{\mu_{2}}=1$ & $\frac{\mu_{1}}{\mu_{2}}=2$ \\
\hline \multirow{9}{*}{$\begin{array}{l}\text { Function } 2 \\
\text { (linear) }\end{array}$} & \multirow[t]{3}{*}{$\frac{\alpha}{\gamma}=3$} & Opt: & 26.037 & 25.778 & 23.483 & 20.369 & 20.450 & 20.670 & 21.261 & 21.779 & 22.439 \\
\hline & & Heur: & 26.106 & 25.847 & 23.552 & 20.536 & 20.617 & 20.833 & 21.415 & 21.924 & 22.575 \\
\hline & & $\%$ Diff: & $0.26 \%$ & $0.27 \%$ & $0.29 \%$ & $0.82 \%$ & $0.81 \%$ & $0.79 \%$ & $0.72 \%$ & $0.67 \%$ & $0.61 \%$ \\
\hline & \multirow{3}{*}{$\frac{\alpha}{\gamma}=5$} & Opt: & 19.661 & 19.479 & 18.539 & 17.079 & 17.191 & 17.381 & 17.957 & 18.491 & 19.112 \\
\hline & & Heur: & 20.053 & 19.849 & 18.933 & 17.482 & 17.575 & 17.765 & 18.331 & 18.851 & 19.464 \\
\hline & & $\%$ Diff: & $1.99 \%$ & $1.90 \%$ & $2.13 \%$ & $2.36 \%$ & $2.23 \%$ & $2.21 \%$ & $2.09 \%$ & $1.95 \%$ & $1.84 \%$ \\
\hline & \multirow[t]{3}{*}{$\frac{\alpha}{\gamma}=10$} & Opt: & 18.220 & 18.038 & 17.377 & 16.335 & 16.444 & 16.641 & 17.208 & 17.741 & 18.344 \\
\hline & & Heur: & 18.773 & 18.577 & 17.937 & 16.740 & 16.843 & 17.029 & 17.606 & 18.142 & 18.760 \\
\hline & & \% Diff: & $3.03 \%$ & $2.99 \%$ & $3.22 \%$ & $2.48 \%$ & $2.42 \%$ & $2.33 \%$ & $2.31 \%$ & $2.26 \%$ & $2.27 \%$ \\
\hline \multirow{9}{*}{$\begin{array}{l}\text { Function } 3 \\
\text { (concave) }\end{array}$} & \multirow[t]{3}{*}{$\frac{\alpha}{\gamma}=3$} & Opt: & 33.459 & 33.211 & 30.984 & 27.864 & 27.953 & 28.167 & 28.753 & 29.277 & 29.926 \\
\hline & & Heur: & 33.467 & 33.219 & 31.024 & 27.989 & 28.075 & 28.280 & 28.862 & 29.384 & 30.025 \\
\hline & & $\%$ Diff: & $0.02 \%$ & $0.02 \%$ & $0.13 \%$ & $0.45 \%$ & $0.44 \%$ & $0.40 \%$ & $0.38 \%$ & $0.36 \%$ & $0.33 \%$ \\
\hline & \multirow[t]{3}{*}{$\frac{\alpha}{\gamma}=5$} & Opt: & 27.352 & 27.183 & 26.240 & 24.613 & 24.729 & 24.904 & 25.451 & 25.985 & 26.579 \\
\hline & & Heur: & 27.685 & 27.504 & 26.583 & 24.817 & 24.932 & 25.102 & 25.673 & 26.217 & 26.815 \\
\hline & & \% Diff: & $1.22 \%$ & $1.18 \%$ & $1.31 \%$ & $0.83 \%$ & $0.82 \%$ & $0.79 \%$ & $0.87 \%$ & $0.89 \%$ & $0.89 \%$ \\
\hline & \multirow[t]{3}{*}{$\frac{\alpha}{\gamma}=10$} & Opt: & 25.870 & 25.702 & 25.058 & 23.546 & 23.662 & 23.831 & 24.349 & 24.871 & 25.452 \\
\hline & & Heur: & 26.222 & 26.052 & 25.360 & 23.926 & 24.045 & 24.211 & 24.780 & 25.323 & 25.898 \\
\hline & & \% Diff: & $1.36 \%$ & $1.36 \%$ & $1.20 \%$ & $1.61 \%$ & $1.62 \%$ & $1.59 \%$ & $1.77 \%$ & $1.82 \%$ & $1.75 \%$ \\
\hline \multirow{9}{*}{$\begin{array}{l}\text { Function } 4 \\
\text { (general) }\end{array}$} & \multirow[t]{3}{*}{$\frac{\alpha}{\gamma}=3$} & Opt: & 26.084 & 25.828 & 23.610 & 20.653 & 20.731 & 20.947 & 21.523 & 22.036 & 22.684 \\
\hline & & Heur: & 26.097 & 25.842 & 23.689 & 20.766 & 20.841 & 21.056 & 21.633 & 22.136 & 22.773 \\
\hline & & \% Diff: & $0.05 \%$ & $0.05 \%$ & $0.33 \%$ & $0.55 \%$ & $0.53 \%$ & $0.52 \%$ & $0.51 \%$ & $0.45 \%$ & $0.40 \%$ \\
\hline & \multirow{3}{*}{$\frac{\alpha}{\gamma}=5$} & Opt: & 19.848 & 19.665 & 18.761 & 17.401 & 17.505 & 17.693 & 18.245 & 18.769 & 19.372 \\
\hline & & Heur: & 20.327 & 20.127 & 19.204 & 17.756 & 17.852 & 18.046 & 18.625 & 19.143 & 19.724 \\
\hline & & $\%$ Diff: & $2.41 \%$ & $2.35 \%$ & $2.36 \%$ & $2.04 \%$ & $1.98 \%$ & $1.99 \%$ & $2.08 \%$ & $1.99 \%$ & $1.82 \%$ \\
\hline & \multirow[t]{3}{*}{$\frac{\alpha}{\gamma}=10$} & Opt: & 18.399 & 18.218 & 17.599 & 16.607 & 16.723 & 16.905 & 17.439 & 17.948 & 18.523 \\
\hline & & Heur: & 18.810 & 18.620 & 18.043 & 16.873 & 16.979 & 17.155 & 17.721 & 18.225 & 18.787 \\
\hline & & $\%$ Diff: & $2.24 \%$ & $2.21 \%$ & $2.52 \%$ & $1.60 \%$ & $1.53 \%$ & $1.48 \%$ & $1.62 \%$ & $1.55 \%$ & $1.43 \%$ \\
\hline
\end{tabular}

definitely promising, if they do not work well in a particular instance then the idea of smoothing the optimal policy could be explored further.

In conclusion, our numerical study yields three major observations. First, the difference in the average costs between operating with a fixed number of servers and operating under the optimal policies for the unrestricted, controlled capacity reduction and uncontrolled capacity reduction cases can be significant. Second, our 2-level heuristic provides a simple, easily implementable alternative to optimal policies without (in most cases) a significant increase in the average costs. This observation seems to be robust in each of the model parameters. Finally, our numerical study supports our unproven conjecture that a sufficient condition for the optimal capacity increase/decrease policy to be monotone is that the worker cost function is increasing and convex. When the convexity assumption is relaxed, examples show that the optimal policy need not be monotone. 
Table 8

Results (optimal average cost, cost of the best 2-level heuristic, and percent difference) of the numerical study for the controlled reduction case of the base parameters restricted to $\gamma=.1$ with various worker cost functions.

\begin{tabular}{|c|c|c|c|c|c|c|c|c|c|c|c|}
\hline & & & \multicolumn{3}{|c|}{$\frac{h_{2}}{h_{1}}=.5$} & \multicolumn{3}{|c|}{$\frac{h_{2}}{h_{1}}=2$} & \multicolumn{3}{|c|}{$\frac{h_{2}}{h_{1}}=5$} \\
\hline & & & $\frac{\mu_{1}}{\mu_{2}}=.5$ & $\frac{\mu_{1}}{\mu_{2}}=1$ & $\frac{\mu_{1}}{\mu_{2}}=2$ & $\frac{\mu_{1}}{\mu_{2}}=.5$ & $\frac{\mu_{1}}{\mu_{2}}=1$ & $\frac{\mu_{1}}{\mu_{2}}=2$ & $\frac{\mu_{1}}{\mu_{2}}=.5$ & $\frac{\mu_{1}}{\mu_{2}}=1$ & $\frac{\mu_{1}}{\mu_{2}}=2$ \\
\hline \multirow{9}{*}{$\begin{array}{l}\text { Function } 2 \\
\text { (linear) }\end{array}$} & \multirow{3}{*}{$\alpha=.3$} & Opt: & 17.433 & 17.273 & 16.550 & 15.560 & 15.669 & 15.805 & 16.288 & 16.750 & 17.224 \\
\hline & & Heur: & 17.596 & 17.448 & 16.730 & 15.710 & 15.811 & 15.930 & 16.370 & 16.798 & 17.250 \\
\hline & & \% Diff: & $0.93 \%$ & $1.01 \%$ & $1.09 \%$ & $0.96 \%$ & $0.90 \%$ & $0.79 \%$ & $0.50 \%$ & $0.29 \%$ & $0.15 \%$ \\
\hline & \multirow[t]{3}{*}{$\alpha=.5$} & Opt: & 16.594 & 16.473 & 15.871 & 14.645 & 14.744 & 14.859 & 15.278 & 15.680 & 16.100 \\
\hline & & Heur: & 16.726 & 16.615 & 16.006 & 14.669 & 14.765 & 14.874 & 15.283 & 15.681 & 16.100 \\
\hline & & \% Diff: & $0.80 \%$ & $0.86 \%$ & $0.85 \%$ & $0.17 \%$ & $0.14 \%$ & $0.10 \%$ & $.03 \%$ & $0.01 \%$ & $0.00 \%$ \\
\hline & \multirow[t]{3}{*}{$\alpha=1$} & Opt: & 15.030 & 14.936 & 14.576 & 13.795 & 13.879 & 13.974 & 14.334 & 14.682 & 15.053 \\
\hline & & Heur: & 15.050 & 14.955 & 14.590 & 13.795 & 13.879 & 13.974 & 14.334 & 14.682 & 15.053 \\
\hline & & \% Diff: & $0.13 \%$ & $0.12 \%$ & $0.10 \%$ & $0.00 \%$ & $0.00 \%$ & $0.00 \%$ & $00 \%$ & $.00 \%$ & $00 \%$ \\
\hline \multirow{9}{*}{$\begin{array}{l}\text { Function } 3 \\
\text { (concave) }\end{array}$} & \multirow[t]{3}{*}{$\alpha=.3$} & Opt: & 23.642 & 23.535 & 22.597 & 20.544 & 20.653 & 20.753 & 1.172 & 21.592 & 22.009 \\
\hline & & Heur: & 23.642 & 23.535 & 22.597 & 20.593 & 20.711 & 20.813 & .253 & 21.698 & 22.134 \\
\hline & & \% Diff: & $0.00 \%$ & $0.00 \%$ & $0.00 \%$ & $0.24 \%$ & $0.28 \%$ & $0.29 \%$ & $39 \%$ & $0.49 \%$ & $0.57 \%$ \\
\hline & \multirow{3}{*}{$\alpha=.5$} & Opt: & 21.155 & 21.062 & 20.436 & 18.922 & 19.015 & 19.103 & 9.466 & 19.828 & 20.191 \\
\hline & & Heur: & 21.155 & 21.062 & 20.436 & 19.099 & 19.213 & 19.304 & 19.713 & 20.128 & 20.530 \\
\hline & & \% Diff: & $0.00 \%$ & $0.00 \%$ & $0.00 \%$ & $0.94 \%$ & $1.04 \%$ & $1.05 \%$ & $27 \%$ & $1.52 \%$ & $1.68 \%$ \\
\hline & \multirow{3}{*}{$\alpha=1$} & Opt: & 18.730 & 18.645 & 18.231 & 17.132 & 17.210 & 17.288 & 17.600 & 17.907 & 18.223 \\
\hline & & Heur: & 18.734 & 18.656 & 18.274 & 17.478 & 17.580 & 17.658 & 8.018 & 18.383 & 18.737 \\
\hline & & $\%$ Diff: & $0.02 \%$ & $0.06 \%$ & $0.23 \%$ & $2.02 \%$ & $2.15 \%$ & $2.14 \%$ & $37 \%$ & $2.66 \%$ & $2.82 \%$ \\
\hline \multirow{9}{*}{$\begin{array}{l}\text { Function } 4 \\
\text { (general) }\end{array}$} & \multirow[t]{3}{*}{$\alpha=.3$} & Opt: & 18.035 & 17.890 & 17.145 & 15.528 & 15.632 & 15.762 & 16.230 & 16.681 & 17.163 \\
\hline & & Heur: & 18.192 & 18.031 & 17.254 & 15.595 & 15.697 & 15.826 & 16.289 & 16.736 & 17.215 \\
\hline & & $\%$ Diff: & $0.87 \%$ & $0.79 \%$ & $0.64 \%$ & $0.43 \%$ & $0.42 \%$ & $0.41 \%$ & $0.37 \%$ & $0.33 \%$ & $0.30 \%$ \\
\hline & \multirow[t]{3}{*}{$\alpha=.5$} & Opt: & 16.562 & 16.433 & 15.786 & 14.493 & 14.592 & 14.712 & 15.147 & 15.567 & 16.016 \\
\hline & & Heur: & 16.722 & 16.589 & 15.873 & 14.518 & 14.615 & 14.737 & 15.177 & 15.600 & 16.056 \\
\hline & & \% Diff: & $0.97 \%$ & $0.95 \%$ & $0.55 \%$ & $0.17 \%$ & $0.16 \%$ & $0.17 \%$ & $0.19 \%$ & $0.21 \%$ & $0.25 \%$ \\
\hline & \multirow[t]{3}{*}{$\alpha=1$} & Opt: & 14.885 & 14.775 & 14.360 & 13.550 & 13.640 & 13.751 & 14.150 & 14.533 & 14.945 \\
\hline & & Heur: & 15.021 & 14.897 & 14.416 & 13.570 & 13.658 & 13.772 & 14.177 & 14.564 & 14.987 \\
\hline & & \% Diff: & $0.91 \%$ & $0.83 \%$ & $0.39 \%$ & $0.15 \%$ & $0.14 \%$ & $0.16 \%$ & $0.19 \%$ & $0.22 \%$ & $0.28 \%$ \\
\hline
\end{tabular}

Table 9

Results (optimal average cost, cost of the best 2-level heuristic, and percent difference) of the numerical study for the unrestricted case of the base parameters restricted to $\gamma=.1$ with various worker cost functions.

\begin{tabular}{|c|c|c|c|c|c|c|c|c|c|c|}
\hline & & \multicolumn{3}{|c|}{$\frac{h_{2}}{h_{1}}=.5$} & \multicolumn{3}{|c|}{$\frac{h_{2}}{h_{1}}=2$} & \multicolumn{3}{|c|}{$\frac{h_{2}}{h_{1}}=5$} \\
\hline & & $\frac{\mu_{1}}{\mu_{2}}=.5$ & $\frac{\mu_{1}}{\mu_{2}}=1$ & $\frac{\mu_{1}}{\mu_{2}}=2$ & $\frac{\mu_{1}}{\mu_{2}}=.5$ & $\frac{\mu_{1}}{\mu_{2}}=1$ & $\frac{\mu_{1}}{\mu_{2}}=2$ & $\frac{\mu_{1}}{\mu_{2}}=.5$ & $\frac{\mu_{1}}{\mu_{2}}=1$ & $\frac{\mu_{1}}{\mu_{2}}=2$ \\
\hline \multirow{3}{*}{$\begin{array}{l}\text { Function } 2 \\
\text { (linear) }\end{array}$} & Opt: & 12.815 & 12.750 & 12.646 & 12.574 & 12.625 & 12.685 & 12.907 & 13.125 & 13.352 \\
\hline & Heur: & 12.815 & 12.750 & 12.646 & 12.574 & 12.625 & 12.685 & 12.907 & 13.125 & 13.352 \\
\hline & \% Diff: & $0.00 \%$ & $0.00 \%$ & $0.00 \%$ & $0.00 \%$ & $0.00 \%$ & $0.00 \%$ & $0.00 \%$ & $0.00 \%$ & $0.00 \%$ \\
\hline \multirow{3}{*}{$\begin{array}{l}\text { Function } 3 \\
\text { (concave) }\end{array}$} & Opt: & 13.062 & 12.997 & 12.893 & 12.822 & 12.872 & 12.933 & 13.155 & 13.372 & 13.599 \\
\hline & Heur: & 13.062 & 12.997 & 12.893 & 12.822 & 12.872 & 12.933 & 13.155 & 13.372 & 13.599 \\
\hline & $\%$ Diff: & $0.00 \%$ & $0.00 \%$ & $0.00 \%$ & $0.00 \%$ & $0.00 \%$ & $0.00 \%$ & $0.00 \%$ & $0.00 \%$ & $0.00 \%$ \\
\hline \multirow{3}{*}{$\begin{array}{l}\text { Function } 4 \\
\text { (general) }\end{array}$} & Opt: & 12.431 & 12.341 & 12.176 & 12.026 & 12.096 & 12.188 & 12.478 & 12.774 & 13.091 \\
\hline & Heur: & 12.611 & 12.500 & 12.267 & 12.056 & 12.125 & 12.222 & 12.556 & 12.875 & 13.222 \\
\hline & \% Diff: & $1.45 \%$ & $1.29 \%$ & $0.74 \%$ & $0.25 \%$ & $0.24 \%$ & $0.28 \%$ & $0.62 \%$ & $0.79 \%$ & $1.00 \%$ \\
\hline
\end{tabular}




\section{Conclusions}

In this paper we considered several models for a potentially temporary, flexible workforce in a dynamic environment. We characterized the structure of the optimal allocation policy of flexible capacity in a tandem queueing system when the capacity is available only for a random amount of time. Furthermore, when the decision-maker has complete control over the capacity available in the system, we proved the intuitive result that the optimal number of workers is non-decreasing in the number of customers at each station. Each of these results stand to simplify both computation and analysis.

We remind the reader that we have assumed that workers can collaborate on a single job. When this does not hold, we believe that the allocation results of Section 3 no longer hold, at least not in both directions. Indeed, even when the capacity is not variable, only the direction leading to the optimality of a policy that is exhaustive in queue 1 holds (see the example in Ahn et al. [1]). We believe that a similar result holds here. Moreover, we do not believe that the assumption that $\mu_{1}=\mu_{2}$ is required for Theorem 4.2, but have been unable to prove it without this assumption.

As of yet we have been unable to prove any analogous results for the capacity increase/decrease decisions in the controlled and uncontrolled capacity reduction cases. While some examples that we considered exhibit the monotonicity, the result does not extend to the case where the opportunity cost per unit time is increasing in the number of servers (as demonstrated by Example 2.4). However, our extensive numerical studies reinforced our conjecture that the optimal capacity increase/decrease policy is monotone in the number of workers when the worker cost function is increasing and convex; this also remains to be proven. Along with this study, we note that other future research directions include a similar analysis for a network of stations and those with capacity that is available for a fixed amount of time.

\section{Acknowledgments}

The authors would like to thank James R. Lewis of Sverdrup Technologies for his help in developing several earlier versions of the model. We would also like to thank the anonymous referees and Robert D. Foley of The Georgia Institute of Technology for their useful comments. The work of the first and third author is supported by the National Science Foundation, NSF-DMI-0132811. The work of the second author is partially supported by the National Science Foundation, NSF-DMI-0245382.

\section{Appendix}

Structure of value functions for finite horizon problems

Proof of Lemma 4.3. Suppose $2 h_{2} \geq h_{1}$. By induction. The result holds trivially for $n=0$. Assume that it holds for $n-1$. Let $d_{n}(i, j)$ denote the optimal number of workers 
in state $(i, j)$ at stage $n$. We show that each inequality holds for $H_{\beta}^{U} v_{n-1}^{U}$. The result then follows from the definition of $v_{n}^{U}$ in (2.7). For the first inequality, choose the actions $d_{n}(i+1, j)$ and $d_{n}(i, j-1)$ in states $(i, j)$ and $(i+1, j-1)$, respectively. For $j \geq 2$

$$
\begin{aligned}
\Delta_{i} H_{\beta}^{U} v_{n-1}^{U}(i, j) \geq & \lambda \Delta_{i} v_{n-1}^{U}(i+1, j)+d_{n}(i+1, j) \mu \Delta_{i} v_{n-1}^{U}(i, j-1) \\
& +\left[1-\left(\lambda+d_{n}(i+1, j) \mu\right)\right] \Delta_{i} v_{n-1}^{U}(i, j),
\end{aligned}
$$

and

$$
\begin{aligned}
\Delta_{i} H_{\beta}^{U} v_{n-1}^{U}(i, j-1) \leq & \lambda \Delta_{i} v_{n-1}^{U}(i+1, j-1)+d_{n}(i, j-1) \mu \Delta_{i} v_{n-1}^{U}(i, j-2) \\
& +\left[1-\left(\lambda+d_{n}(i, j-1) \mu\right)\right] \Delta_{i} v_{n-1}^{U}(i, j-1) .
\end{aligned}
$$

Taking the difference we have

$$
\begin{aligned}
\Delta_{i} & H_{\beta}^{U} v_{n-1}^{U}(i, j-1)-\Delta_{i} H_{\beta}^{U} v_{n-1}^{U}(i, j) \\
\leq & \lambda\left[\Delta_{i} v_{n-1}^{U}(i+1, j-1)-\Delta_{i} v_{n-1}^{U}(i+1, j)\right] \\
& +d_{n}(i, j-1) \mu\left[\Delta_{i} v_{n-1}^{U}(i, j-2)-\Delta_{i} v_{n-1}^{U}(i, j-1)\right] \\
& +\left[1-\left(\lambda+d_{n}(i+1, j) \mu\right)\right]\left[\Delta_{i} v_{n-1}^{U}(i, j-1)-\Delta_{i} v_{n-1}^{U}(i, j)\right] .
\end{aligned}
$$

The inductive hypothesis yields the first assertion. In the case that $j=1$ and $i \geq 1$, a similar argument yields

$$
\begin{aligned}
\Delta_{i} & H_{\beta}^{U} v_{n-1}^{U}(i, 0)-\Delta_{i} H_{\beta}^{U} v_{n-1}^{U}(i, 1) \\
\leq & \lambda\left[\Delta_{i} v_{n-1}^{U}(i+1,0)-\Delta_{i} v_{n-1}^{U}(i+1,1)\right] \\
& +d_{n}(i, 0) \mu\left[\Delta_{i} v_{n-1}^{U}(i-1,1)-\Delta_{i} v_{n-1}^{U}(i, 0)\right] \\
& +\left[1-\left(\lambda+d_{n}(i+1,1) \mu\right)\right]\left[\Delta_{i} v_{n-1}^{U}(i, 0)-\Delta_{i} v_{n-1}^{U}(i, 1)\right] .
\end{aligned}
$$

The result now follows directly from the inductive hypothesis. If $j=1$ and $i=0$, we first note that $d_{n}(0,0)=0$ is an optimal action (otherwise workers would be idle). Thus, the previous inequality holds with the second term equal to zero. $j \geq 1$

For the second inequality, let $\Delta_{j}^{2} g(i, j) \equiv \Delta_{j} g(i, j+1)-\Delta_{j} g(i, j)$. Note for

$$
\begin{aligned}
\Delta_{j}^{2} H_{\beta}^{U} v_{n-1}^{U}(i, j) \geq & \lambda \Delta_{j}^{2} v_{n-1}^{U}(i, j)+d(i, j) \mu \Delta_{j}^{2} v_{n-1}^{U}(i, j-1) \\
& +[1-(\lambda+d(i, j+2) \mu)] \Delta_{j}^{2} v_{n-1}^{U}(i, j),
\end{aligned}
$$

and for $j=0$ and $i \geq 1$

$$
\begin{aligned}
\Delta_{j}^{2} H_{\beta}^{U} v_{n-1}^{U}(i, 0) \geq & \lambda \Delta_{j}^{2} v_{n-1}^{U}(i, 0)+d(i, 0) \mu\left[\Delta_{j} v_{n-1}^{U}(i, 0)-\left(v_{n-1}^{U}(i, 0)-v_{n-1}^{U}(i-1,1)\right)\right] \\
& +[1-(\lambda+d(i, j+2) \mu)] \Delta_{j}^{2} v_{n-1}^{U}(i, j) .
\end{aligned}
$$


The result follows by the inductive hypothesis. The case for $i=j=0$ is analogous to the previous argument.

The third inequality yields

$$
\begin{aligned}
\Delta_{i} & H_{\beta}^{U} v_{n-1}^{U}(i-1,1)-\Delta_{i} H_{\beta}^{U} v_{n-1}^{U}(i, 0) \\
\leq & \lambda\left[\Delta_{i} v_{n-1}^{U}(i, 1)-\Delta_{i} v_{n-1}^{U}(i+1,0)\right] \\
& +d_{n}(i, 1) \mu\left[\Delta_{i} v_{n-1}^{U}(i-1,0)-\Delta_{i} v_{n-1}^{U}(i-1,1)\right] \\
& +\left[1-\left(\lambda+d_{n}(i, 0) \mu\right)\right]\left[\Delta_{i} v_{n-1}^{U}(i-1,1)-\Delta_{i} v_{n-1}^{U}(i, 0)\right] .
\end{aligned}
$$

Each term is non-positive by the inductive hypothesis by noting

$$
\Delta_{i} v_{n-1}^{U}(i-1,0)-\Delta_{i} v_{n-1}^{U}(i-1,1)=\Delta_{j} v_{n-1}^{U}(i-1,0)-\Delta_{j} v_{n-1}^{U}(i, 0) .
$$

To show the fourth inequality choose $d_{n}(i, 1)$ and $d_{n}(i-1,1)$ for the two processes starting in $(i, 0)$,

$$
\begin{aligned}
\Delta_{j} & H_{\beta}^{U} v_{n-1}^{U}(i, 0)-\left[H_{\beta}^{U} v_{n-1}^{U}(i, 0)-H_{\beta}^{U} v_{n-1}^{U}(i-1,1)\right] \\
\geq & \lambda\left[\Delta_{j} v_{n-1}^{U}(i+1,0)-\left[v_{n-1}^{U}(i+1,0)-v_{n-1}^{U}(i, 1)\right]\right] \\
& +\left[1-\left(\lambda+d_{n}(i, 1) \mu\right)\right]\left[\Delta_{j} v_{n-1}^{U}(i, 0)-\left[v_{n-1}^{U}(i, 0)-v_{n-1}^{U}(i-1,1)\right]\right] \\
& +d_{n}(i-1,1) \mu\left[\left[v_{n-1}^{U}(i, 0)-v_{n-1}^{U}(i-1,1)\right]-\Delta_{j} v_{n-1}^{U}(i-1,0)\right] .
\end{aligned}
$$

Similarly,

$$
\begin{aligned}
H_{\beta}^{U} & v_{n-1}^{U}(i, 0)-H_{\beta}^{U} v_{n-1}^{U}(i-1,1)-\Delta_{j} H_{\beta}^{U} v_{n-1}^{U}(i-1,0) \\
\geq & \lambda\left[v_{n-1}^{U}(i+1,0)-v_{n-1}^{U}(i, 1)-\Delta_{j} v_{n-1}^{U}(i, 0)\right] \\
& +\left[1-\left(\lambda+d_{n}(i, 0) \mu\right)\right]\left[v_{n-1}^{U}(i, 0)-v_{n-1}^{U}(i-1,1)-\Delta_{j} v_{n-1}^{U}(i-1,0)\right] \\
& +d_{n}(i-1,0) \mu\left[\Delta_{j} v_{n-1}^{U}(i-1,0)-\left[v_{n-1}^{U}(i-1,0)-v_{n-1}^{U}(i-2,1)\right]\right] .
\end{aligned}
$$

In either case, the inductive hypothesis yields the result for $H_{\beta}^{U} v_{n-1}^{U}$. Recalling that $2 h_{2} \geq h_{1}$ yields the result for $v_{n}^{U}$.

Suppose now that $h_{1}>2 h_{2}$. Again, the results hold for $n=0$ trivially. Assume that it is true for $n-1$. To prove the first inequality, choose potentially sub-optimal actions to get for $i \geq 1$

$$
\begin{aligned}
\Delta_{j} & v_{n}^{U}(i, j)-\Delta_{j} v_{n}^{U}(i, j+1) \\
\leq & \lambda\left[\Delta_{j} v_{n-1}^{U}(i+1, j)-\Delta_{j} v_{n-1}^{U}(i+1, j+1)\right] \\
& +d_{n}(i, j) \mu\left[\Delta_{j} v_{n-1}^{U}(i-1, j+1)-\Delta_{j} v_{n-1}^{U}(i-1, j+2)\right] \\
& +\left[1-\left(\lambda+d_{n}(i, j+2) \mu\right)\right]\left[\Delta_{j} v_{n-1}^{U}(i, j)-\Delta_{j} v_{n-1}^{U}(i, j+1)\right],
\end{aligned}
$$


and for $i=0$

$$
\begin{aligned}
\Delta_{j} & v_{n}^{U}(0, j)-\Delta_{j} v_{n}^{U}(0, j+1) \\
\leq & \lambda\left[\Delta_{j} v_{n-1}^{U}(1, j)-\Delta_{j} v_{n-1}^{U}(1, j+1)\right] \\
& +d_{n}(0, j) \mu\left[\Delta_{j} v_{n-1}^{U}(0, j-1)-\Delta_{j} v_{n-1}^{U}(0, j)\right] \\
& +\left[1-\left(\lambda+d_{n}(0, j+2) \mu\right)\right]\left[\Delta_{j} v_{n-1}^{U}(0, j)-\Delta_{j} v_{n-1}^{U}(0, j+1)\right] .
\end{aligned}
$$

Similarly, for the second inequality for $i \geq 2$

$$
\begin{aligned}
\Delta_{j} & v_{n}^{U}(i-1, j+1)-\Delta_{j} v_{n}^{U}(i, j) \\
\leq & \lambda\left[\Delta_{j} v_{n-1}^{U}(i, j+1)-\Delta_{j} v_{n-1}^{U}(i+1, j)\right] \\
& +d_{n}(i-1, j+1) \mu\left[\Delta_{j} v_{n-1}^{U}(i-2, j+2)-\Delta_{j} v_{n-1}^{U}(i-1, j+1)\right] \\
& +\left[1-\left(\lambda+d_{n}(i, j+1) \mu\right)\right]\left[\Delta_{j} v_{n-1}^{U}(i-1, j+1)-\Delta_{j} v_{n-1}^{U}(i, j)\right],
\end{aligned}
$$

and for $i=1$

$$
\begin{aligned}
\Delta_{j} & v_{n}^{U}(0, j+1)-\Delta_{j} v_{n}^{U}(1, j) \\
\leq & \lambda\left[\Delta_{j} v_{n-1}^{U}(1, j+1)-\Delta_{j} v_{n-1}^{U}(2, j)\right] \\
& +d_{n}(0, j+1) \mu\left[\Delta_{j} v_{n-1}^{U}(0, j)-\Delta_{j} v_{n-1}^{U}(0, j+1)\right] \\
& +\left[1-\left(\lambda+d_{n}(1, j+1) \mu\right)\right]\left[\Delta_{j} v_{n-1}^{U}(0, j+1)-\Delta_{j} v_{n-1}^{U}(1, j)\right] .
\end{aligned}
$$

In each case, the induction hypothesis proves the result.

\section{Stability results}

We provide conditions under which a policy yields a stable Markov process. This leads to a guarantee that the process also lends finite average cost, and thus to the existence of a finite solution to the ACOE. The first result states conditions for stability for the unrestricted and controlled capacity reduction cases.

Proposition A.1. Suppose for some $k^{*} \in\{0,1, \ldots, \ell\}$

$$
\frac{\lambda}{\mu_{1}}+\frac{\lambda}{\mu_{2}}<k^{*} \text {. }
$$

Then in either the unrestricted or controlled capacity reduction models, there exists a policy $\pi$ such that $\rho^{\pi}<\infty$.

Proof. In either case, one may choose the policy that has $k^{*}$ workers available for all but a finite amount of time. The system is then the same system studied for 2 workers 
in Ahn et al. [1] where the average holding costs is shown to be finite. The $k^{*}$ worker case is directly analogous. Adding $r\left(k^{*}\right)$ to this finite amount yields the result.

When the capacity reduction is uncontrolled, the workforce cannot be made constant and the argument in Proposition A.1 does not carry over. For the remainder of this section we study this problem and thus, suppress the superscript $F$ on all quantities. Note that this no longer means that quantities apply to all three models. Since the workers are flexible, the system can be viewed as a reentrant line with one common pool of resources that can be split amongst two classes - stage 1 and stage 2 customers. Stability and convergence of moments results for standard reentrant lines (with fixed service capacity) can be obtained using fluid limit techniques as in Dai [11] and Dai and Meyn [12]. We adapt these results to our setting with a varying service capacity. In general, the fluid limit results are very useful in analyzing network stability even when arrival and service times follow a general distribution. This is not taken advantage of here; only exponential times are addressed. What is made use of is the fact that the results go beyond stability in a positive recurrence sense and ensure finite average queue length.

Fix a Markovian head of the line (HL) policy, $\pi$. That is, $\pi$ is such that workers can collaborate to work on only the first customers in each of the queues and worker allocations remain constant in between changes in the Markovian state $X(t)=(Q(t), K(t))$, where $Q(t)=\left(Q_{1}(t), Q_{2}(t)\right)$ is the queue length process (including the customers in service) and $K(t)$ is the number of workers available at time $t$.

We explore the statistical regularity of this policy through fluid scaling, a law of large numbers type scaling of space and time. Define the norm

$$
|X(t)| \equiv Q_{1}(t)+Q_{2}(t)+K(t)
$$

and consider the scaled process

$$
\bar{Q}^{x}(t) \equiv \frac{1}{|x|} Q^{x}(|x| t),
$$

where the superscript denotes the dependence on the initial state $x=X(0)$. Letting $|x| \rightarrow \infty$, any limit point $\bar{Q}(t)$ is called a fluid limit of the queue length process. We will show that every fluid limit is a solution to a set of equations known as the fluid model. The fluid model is said to be stable if there exists a fixed time $t_{0}$ such that $\bar{Q}(t)=0$ for all $t \geq t_{0}$. That is, the fluid model is stable if all queues eventually drain and once drained stay empty.

Let the customer inter-arrival times be $\xi(n), n=1,2, \ldots$, and the service requirements at stage $m$ be $\eta_{m}(n), n=1,2, \ldots ; m=1,2$. Recall that we have assumed that $\xi$ and $\eta_{m}$ are sequences of i.i.d. exponential random variables with means $1 / \lambda$ and $1 / \mu_{m}$ respectively. Let

$$
\begin{aligned}
E(t) & =\max \{n \geq 0: \xi(1)+\xi(2)+\cdots+\xi(n-1) \leq t\}, \quad t \geq 0, \\
S_{m}(t) & =\max \left\{n \geq 0: \eta_{m}(1)+\eta_{m}(2)+\cdots+\eta_{m}(n-1) \leq t\right\}, \quad t \geq 0,
\end{aligned}
$$


where the maximum of an empty set is zero. $E(t)$ is the total number of arrivals by time $t$. $S_{m}(t)$ represents the total number of service completions from station $m$ given $t$ units of work. We make the following assumptions on $\pi$ regarding capacity increases.

A1. (i) $\pi$ does not reject any arriving workers up to capacity $\ell$. (ii) Workers are never idle while customers are present.

Under A1, it is easy to see that the number of workers available follows a birth-death process with death rates $k \gamma$ and birth rates $\alpha(k), k=0,1, \ldots, \ell$. Such a process has steady-state probabilities $p_{k}$ given in Proposition 2.2. Hence, the average number of workers available in steady state is

$$
z=\sum_{k=1}^{\ell}\left(k p_{k}\right)
$$

Let $Y_{k}^{x}(t)$ be the cumulative time there are exactly $k$ hired workers available in $[0, t)$ given the initial state $x$. Suppose $W_{m k}^{x}(t)$ is the cumulative amount of work done by $t$ on stage $m$ customers during the times there are exactly $k$ workers available. Thus, $S_{m}\left(\sum_{k=0}^{\ell} W_{m k}^{x}(t)\right)$ is the number of completions from stage $m$ by $t$. Let $I_{k}^{x}(t)$ be the cumulative amount of idle time while exactly $k$ workers are available so that $T_{k}^{x}(t) \equiv Y_{k}^{x}(t)-I_{k}^{x}(t)$ is the amount of time there are customers to serve in $[0, t)$ when there are exactly $k$ workers available. These processes are all defined on the domain $[0, \infty)$ and are assumed to be right continuous with left limits. The definitions above imply the following system equations:

$$
\begin{aligned}
& Q_{1}^{x}(t)=Q_{1}^{x}(0)+E^{x}(t)-S_{1}^{x}\left(\sum_{k=0}^{\ell} W_{1 k}^{x}(t)\right) \\
& Q_{2}^{x}(t)=Q_{2}^{x}(0)+S_{1}^{x}\left(\sum_{k=0}^{\ell} W_{1 k}^{x}(t)\right)-S_{2}^{x}\left(\sum_{k=0}^{\ell} W_{2 k}^{x}(t)\right) \\
& Q^{x}(t) \geq 0 \\
& \sum_{k=0}^{\ell} Y_{k}^{x}(t)=t \\
& T_{k}^{x}(t)+I_{k}^{x}(t)=Y_{k}^{x}(t), \quad k=0,1, \ldots, \ell \\
& W_{1 k}^{x}(t)+W_{2 k}^{x}(t)=k T_{k}^{x}(t), \quad k=0,1, \ldots, \ell \\
& Y_{k}^{x}(t), T_{k}^{x}(t), I_{k}^{x}(t), W_{1 k}^{x}(t), \text { and } W_{2 k}^{x}(t) \text { are nondecreasing and start from } 0 \\
& \int_{0}^{\infty}\left(Q_{1}^{x}(t)+Q_{2}^{x}(t)\right) d\left(\sum_{k=0}^{\ell} I_{k}^{x}(t)\right)=0
\end{aligned}
$$


Note that (A.9) is the non-idling constraint and guarantees that the idle time increases only if the total queue length is zero.

The next proposition, a variant of Theorem 4.1 in Dai [11], presents the fluid model and establishes convergence of the scaled processes. This convergence is uniform on compact sets (u.o.c.).

Proposition A.2. Consider any Markovian HL policy under A1. The following holds with probability one. For any sequence of initial states $\left\{x_{j}\right\} \in \mathbb{X}$ with $\left|x_{j}\right| \rightarrow \infty$, there exists a subsequence $\left\{x_{i}\right\},\{i\} \subseteq\{j\}$, with $\left|x_{i}\right| \rightarrow \infty$ such that

$$
\begin{aligned}
\left(\bar{Q}^{x_{i}}(0), \bar{K}^{x_{i}}(0)\right) & \rightarrow(\bar{Q}(0), 0), \\
\left(\bar{Q}^{x_{i}}(t), \bar{T}^{x_{i}}(t), \bar{W}^{x_{i}}(t)\right) & \rightarrow(\bar{Q}(t), \bar{T}(t), \bar{W}(t)) \text { u.o.c. }
\end{aligned}
$$

where $(\bar{Q}, \bar{T}, \bar{W})$ satisfies the following set of equations:

$$
\begin{aligned}
& \bar{Q}_{1}(t)=\bar{Q}_{1}(0)+\lambda t-\mu_{1}\left(\sum_{k=0}^{\ell} \bar{W}_{1 k}(t)\right) \\
& \bar{Q}_{2}(t)=\bar{Q}_{2}(0)+\mu_{1}\left(\sum_{k=0}^{\ell} \bar{W}_{1 k}(t)\right)-\mu_{2}\left(\sum_{k=0}^{\ell} \bar{W}_{2 k}(t)\right) \\
& \bar{Q}(t) \geq 0 \\
& \sum_{k=0}^{\ell} p_{k}=1 \\
& \bar{T}_{k}(t)+\bar{I}_{k}(t)=p_{k} t, \quad k=0,1, \ldots, \ell \\
& \bar{W}_{1 k}(t)+\bar{W}_{2 k}(t)=k \bar{T}_{k}(t), \quad k=0,1, \ldots, \ell \\
& \bar{Y}_{k}(t), \bar{T}_{k}(t), \bar{I}_{k}(t), \bar{W}_{1 k}(t), \text { and } \bar{W}_{2 k}(t) \text { are nondecreasing and start from } 0, \\
& \int_{0}^{\infty}\left(\bar{Q}_{1}(t)+\bar{Q}_{2}(t)\right) d\left(\sum_{k=0}^{\ell} \bar{I}_{k}(t)\right)=0
\end{aligned}
$$

Proof. Notice that for $m=1,2$,

$$
\frac{1}{\left|x_{j}\right|} Q_{m}^{x_{j}}(0) \leq 1, \quad \frac{1}{\left|x_{j}\right|} K^{x_{j}}(0) \leq 1,
$$

for all $j$. Also, $0 \leq K^{x_{j}}(0) \leq \ell$. Therefore, there exists a subsequence $\left|x_{i}\right| \rightarrow \infty$ such that (A.10) holds.

For any $0 \leq t_{1} \leq t_{2}$, and each $m$ and $k$, we have

$$
0 \leq \bar{W}_{m k}^{x}\left(t_{2}\right)-\bar{W}_{m k}^{x}\left(t_{1}\right) \leq k\left(\bar{T}_{m k}^{x}\left(t_{2}\right)-\bar{T}_{m k}^{x}\left(t_{1}\right)\right) \leq \ell\left(t_{2}-t_{1}\right) .
$$


That is, $\left\{\bar{T}_{k}^{x}(t),|x| \geq 1\right\}$ and $\left\{\bar{W}_{m k}^{x}(t),|x| \geq 1\right\}$ are uniformly Lipschitz, and hence equicontinuous. Setting $t_{1}=0$ and $t_{2}=t$ yields uniform bounds $0 \leq \bar{T}_{k}^{x}(t) \leq t$ and $0 \leq \bar{W}_{m k}^{x}(t) \leq \ell t$. Therefore, by the Arzelà-Ascoli theorem, any subsequence of $\bar{T}_{k}^{x}(t)$ has a u.o.c. convergent subsequence. Similarly, for $\bar{W}_{m k}^{x}(t)$. The families $\left\{\bar{I}_{k}^{x}(t),|x| \geq 1\right\}$ and $\left\{\bar{Y}_{k}^{x}(t),|x| \geq 1\right\}$ can be shown to have a u.o.c. convergent subsequence in the same manner. Since $K^{x}(t)$ is ergodic, the (functional) Strong Law of Large Numbers (SLLN) (cf. Glynn and Whitt [18]) can be applied to get

$$
\begin{aligned}
\bar{Y}_{k}(t) & =p_{k} t, \\
\bar{E}(t) & =\lambda t, \\
\bar{S}_{m}(t) & =\mu_{m} t .
\end{aligned}
$$

Equations (A.12) and (A.13) then follow from (A.2) and (A.3) where the random time change is valid by Theorem 5.3 of Chen and Yao [9]. Equations (A.14)-(A.18) are a consequence of (A.4)-(A.8) and (A.19) follows from (A.9) and Lemma 4.4 of Dai [11].

The next proposition says that the desired stability results hold if on average the offered workload by the arriving customers is less than the capacity of the hired workers.

Proposition A.3. Suppose $z$ is defined as in Proposition 2.2. If $\lambda\left(1 / \mu_{1}+1 / \mu_{2}\right)<z$, then for any Markovian HL policy $\pi$ satisfying A1, the fluid model is stable and, thus, an invariant probability $\psi$ exists for $X=\{X(t), t \geq 0\}$. Furthermore,

$$
\lim _{t \rightarrow \infty} \mathbb{E}_{x}^{\pi}\left[Q_{m}(t)\right]=\mathbb{E}_{\psi}^{\pi}\left[Q_{m}(0)\right]<\infty, \quad m=1,2,
$$

and $\rho^{\pi}<\infty$.

Proof. To show that the fluid model is stable, consider the immediate workload given by

$$
\begin{aligned}
\bar{L}(t) & \equiv \bar{Q}_{1}(t) / \mu_{1}+\left(\bar{Q}_{1}(t)+\bar{Q}_{2}(t)\right) / \mu_{2} \\
& =\bar{Q}_{1}(0) / \mu_{1}+\left(\bar{Q}_{1}(0)+\bar{Q}_{2}(0)\right) / \mu_{2}+\lambda\left(1 / \mu_{1}+1 / \mu_{2}\right) t-\sum_{k=0}^{\ell}\left(k \bar{T}_{k}(t)\right) .
\end{aligned}
$$

$\bar{L}(t)$ is nonnegative and equals zero if and only if $\bar{Q}_{1}(t)=\bar{Q}_{2}(t)=0$. Because $\bar{T}_{k}(t)$ and $\bar{W}_{m k}(t)$ are Lipschitz continuous, so are $\bar{Q}_{m}(t)$ and $\bar{L}(t)$. Lipschitz continuity implies absolute continuity, so $\bar{L}(t)$ is differentiable almost everywhere. When $\left(\bar{Q}_{1}(t)+\bar{Q}_{2}(t)\right)>0$, (A.19) and (A.16) imply $\dot{\bar{I}}_{k}(t)=0$ and $\dot{\bar{T}}_{k}(t)=p_{k}$, and thus

$$
\dot{\bar{L}}(t)=\lambda\left(1 / \mu_{1}+1 / \mu_{2}\right)-z .
$$

By Lemma 5.2 of Dai [11], $\bar{L}(t) \equiv 0$ for $t \geq\left(z-\lambda\left(1 / \mu_{1}+1 / \mu_{2}\right)\right)^{-1}$. Therefore, the fluid model is stable. 
The results showing that stability of the fluid model implies stability of the original system are proven in Dai [11] and Dai and Meyn [12]. Since $K(t)$ is uniformly bounded, it is easily incorporated into these proofs. The existence of an invariant probability for $X$ follows from Theorem 4.2 of Dai [11]. From Theorem 4.1 of Dai and Meyn [12], we have (A.20). Since the holding cost rates are linear, this together with a bounded worker cost imply $\rho^{\pi}<\infty$.

Note that the above arguments can be carried over without much difficulty to a network of any finite number of queues in tandem. Since this just amounts to more bookkeeping, the details are omitted.

\section{References}

[1] H. Ahn, I. Duenyas and M.E. Lewis, The optimal control of a two-stage tandem queueing system with flexible servers, Probability in the Engineering and Informational Sciences 16(4) (2002) 453-469.

[2] H. Ahn, I. Duenyas and R.Q. Zhang, Optimal stochastic scheduling of a two-stage tandem queue with parallel servers, Advances in Applied Probability 31 (1999) 1095-1117.

[3] S. Andradottir, H. Ayhan and D. Down, Server assignment policies for maximizing the steady-state throughput of finite queueing systems, Management Science 47(10) (October 2001) 1421-1439.

[4] J.J. Bartholdi III, L.A. Bunimovich and D.D. Eisenstein, Dynamics of 2- and 3-worker 'bucket brigade' production lines, Operations Research 47(3) (1999) 488-491.

[5] J.J. Bartholdi III and D.D. Eisenstein, A production line that balances itself, Operations Research 44(1) (1996) 21-34.

[6] J.J. Bartholdi III, D.D. Eisenstein and R.D. Foley, Performance of bucket brigades when work is stochastic, Operations Research 49(5) (2001) 710-719.

[7] D.P. Bertsekas, Dynamic Programming and Optimal Control, volume 2, (Athena Scientific, Belmont, MA, 1995).

[8] P. Brémaud, Point Processes and Queues; Martingale Dynamics (Springer-Verlag, New York, 1981).

[9] H. Chen and D. Yao, Fundamentals of Queueing Networks (Springer-Verlag, New York, 2001).

[10] T.B. Crabill, Optimal control of a service facility with variable exponential service times and constant arrival rate, Management Science 18(9) (May 1972) 560-566.

[11] J. Dai, On positive harris recurrence of multiclass queueing networks: a unified approach via fluid limit models, Annals of Applied Probability 5 (1995) 49-77.

[12] J. Dai and S. Meyn, Stability and convergence of moments for multiclass queueing networks via fluid limit models, IEEE Transactions on Automatic Control 40 (1995) 1889-1904.

[13] I. Duenyas, D. Gupta and T. Olsen, Control of a single server tandem queueing system with setups, Operations Research 46(2) (March-April 1998) 218-230.

[14] A. Federgruen and K. So, Optimal maintenance policies for single-server queuing-systems subject to breakdowns, Operations Research 38(2) (March-April 1990) 330-343.

[15] A. Federgruen and K. So, Optimality of threshold policies in single-server queuing-systems with server vacations, Advances in Applied Probability 23(2) (June 1991) 388-405.

[16] E.A. Feinberg and O. Kella, Optimality of $D$-policies for an $\mathrm{M} / \mathrm{G} / 1$ queue with a removable server, Queueing Systems 42 (2002) 355-376.

[17] J.M. George and J.M. Harrison, Dynamic control of a queue with adjustable service rate, Operations Research 49(5) (September-October 2001) 720-731.

[18] P. Glynn and W. Whitt, Limit theorems for cumulative processes, Stochastic Processes and their Applications 47 (1993) 299-314. 
[19] D. Heyman, Optimal operating policies for M/G/1 queuing systems, Operations Research 16(2) (March-April 1968) 362-382.

[20] S. Iravani, M. Posner and J. Buzacott. A two-stage tandem queue attended by a moving server with holding and switching costs, Queueing Systems 26(2/3) (1997) 203-228.

[21] T. Javidi, N. Song and D. Teneketzis, Expected makespan minimization of identical machines in two interconnected queues: Probability In The Engineering And Informational Sciences 15(4) (2001) 409-443.

[22] S.A. Lippman, Applying a new device in the optimization of exponential queueing system, Operations Research 23(4) (1975) 687-710.

[23] P. Nain, Interchange arguments for classical scheduling problems in queues, Systems and Control Letters 12 (1989) 177-184.

[24] L.I. Sennott, Stochastic Dynamic Programming and the Control of Queueing Systems (Wiley Series in Probability and Statistics. John Wiley and Sons, Inc., New York, 1999).

[25] S. Stidham, Jr and R. Weber, Monotonic and insensitive optimal policies for control of queues with undiscounted costs, Operations Research 37(4) (Jul-Aug 1989) 611-625.

[26] M. Van Oyen, E. Senturk-Gel and W. Hopp, Performance opportunity for workforce agility in collaborative and noncollaborative work systems, IIE Transactions 33(9) (2001) 761-777.

[27] M. Yadin and P. Naor, Queuing-systems with a removable service station, Operational Research Quarterly 14(1) (1963) 393-405. 